\title{
Prediction of kerosene properties at supercritical pressures by Artificial Neural Network
}

\author{
Bo Li ${ }^{1}$ Yachao Lee ${ }^{2}$ Wei Yao ${ }^{3}$ Yang $\mathrm{Lu}^{4}$ and Xuejun Fan ${ }^{5}$ \\ Key Laboratory of High-Temperature Gas Dynamics, Institute of Mechanics, CAS, Beijing \\ 100190, China \\ School of Engineering Science, University of Chinese Academy of Science, Beijing 100049, China
}

\begin{abstract}
The most important advantages of the artificial neural network (ANN) are the flexibility to model the nonlinear problems and the capability to approximate any functions with highdimensional data requiring no detailed information about the system. In this study, three efficient yet accurate ANN models have been successfully developed to predict the fluid properties of China RP3 kerosene at supercritical pressure by applying the ANN method and the principle of Extended Corresponding State (ECS). The analysis shows that the properties of kerosene predicted by the trained ANN models agree well with the calculated results of the ECS by SuperTrapp. The correlation coefficients $(R)$ between ANN prediction and ECS calculation by SuperTrapp are higher than 0.99 , and most relative errors are lower than $0.1 \%$, while the speed of ANN prediction is several orders (arriving at $10^{4}$ ) higher than ECS calculation by SuperTrapp, especially near the critical point. Additionally, the performance of the trained ANN coupling with CFD modelling was analyzed as well in this study and exhibited a good result.
\end{abstract}

\section{Nomenclature}

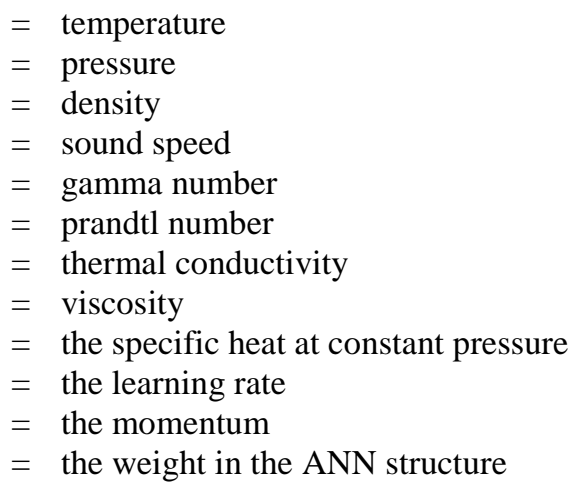

\section{Introduction}

$\mathrm{H}^{2}$

YPERSONIC flight is a trend in the development of modern aerospace technology. Scramjet, which is an ideal power device for hypersonic vehicles, is becoming one of the hot spots in international research. In the development of scramjet, one of the key issues is the thermal protection because the scramjets are usually exposed to an extremely harsh thermal environment due to the flow of sorghum and combustion heat release. Among many

\footnotetext{
${ }^{1}$ PHD student, Institute of Mechanics (CAS), libo2@imech.ac.cn.

${ }^{2}$ PHD student, Institute of Mechanics (CAS), liyachao@imech.ac.cn.

${ }^{3}$ Associate Professor, Institute of Mechanics (CAS), weiyao@imech.ac.cn, AIAA member (Corresponding author).

${ }^{4}$ Associate Professor, Institute of Mechanics (CAS), luyang @imech.ac.cn.

${ }^{5}$ Professor, Institute of Mechanics (CAS), xfan@imech.ac.cn, AIAA lifetime member (Corresponding author).
} 
cooling methods, such as film cooling, transpiration cooling, active cooling, i.e., regeneration cooling with kerosene as coolant has been considered one of the most effective and practical one for scramjets [1,2]. Regeneration cooling means that the fuel firstly flows through the cooling channel as a coolant to absorb heat from high-temperature components, and then is injected into the combustion chamber to burn to release the absorbed heat, so that the heat is "regenerated". Compared with other thermal protection methods, regenerative cooling has many obvious advantages [3]. During regeneration cooling, the fuel not only absorbs heat through the changing of enthalpy using its physical heat sink, but also absorbs heat through the chemical reaction using its chemical heat sink. As a typical hydrocarbon fuel, kerosene is an ideal coolant for its high heat sink, and it has series of other advantages, such as high energy density, small storage footprint, and economy compared to other hydrocarbon fuels.

In the regenerative cooling channel, the working pressure of kerosene is higher than its critical pressure, and the special change of thermal properties under supercritical pressure will induce complex flow and heat transfer phenomena. At present, a fundamental understanding of the relationship between the thermal properties changing and heat transfer characteristics of kerosene is lacking, because though some experimental studies on supercritical kerosene has been conducted, numerical simulations are insufficient due to the complexity of the composition of kerosene. According to the composition and the heat transfer characteristics of China RP3 kerosene, several physical surrogate models, such as Fan's three-component model [4], Cheng's five-component model [5] and Zhong's tencomponent model [6], have been proposed to analyze the regenerative cooling problem. The thermodynamic and transport properties of the kerosene compound can be accurately predicted by the principle of Extended Corresponding State (ECS) [7, 8], which has been embedded in the hydrocarbon property database SuperTrapp [9]. But in threedimensional CFD (computational fluid dynamics) modellings, property calculations based on the ECS method is timeconsuming and resource-wasting, especially around the critical points ( $630 \mathrm{~K}$ and above $2.4 \mathrm{MPa}$ ). It is desirable to develop an efficient yet accurate method to predict the fluid properties of kerosene, which would make CFD modeling coupled with real-gas effects computationally-affordable.

The artificial neural network (ANN), originating from biological neural network (BNN), is one of widely used statistical learning algorithms in recent years and has been successfully applied in function approximation, classification, time series prediction, filtering, data association and optimization [10]. The ANN structure functions like biological neurons with an ability to learn from a set of input-output parameter space it is subjected to, and then, predicted the output for a new sample set at a much high speed and with a sufficient level of accuracy. Usually, the most important advantages of ANN are the flexibility to model the nonlinear problems and the capability to approximate any functions with high-dimensional data requiring no detailed information about the system. And it seem to that ANN has the ability to handle large and complex interrelated parameters by simply ignoring excess data that are of minimal significance and concentrating instead on the more important inputs [11]. Among several types of $\mathrm{ANN}_{\mathrm{S}}$, the back-propagation artificial neural network (BP-ANN) is the most popular one and has have been widely applied $[12,13]$. The main features of BP-ANN are that the weights are adjusted by the information transmitting forward and the errors transferring reversely. However, there is a disadvantage of ANN that it cannot be used to find or explain physical laws involved in a phenomenon, because some mathematical information that contributes to the understanding of physical and thermodynamic comportment can only be obtained by solving equations [14]. Nevertheless ANN is very useful in many fields of knowledge. ANN is a feasible method to improve the calculation efficiency of complex nonlinear problems and has been successfully applied to predict the material properties [12, 1418]. This study will use ANN to predict the fluid properties of kerosene, including the gas state of equation, the fluid phase, the thermodynamic and transport properties, in replacement of the computationally-expansive ECS method.

To improve the calculation efficiency, a map between the environment state and the fluid properties of kerosene is built using an artificial neural network (ANN). A BP-ANN model with three hidden layers is used to estimate the fluid properties of kerosene at supercritical pressures. The developed ANN structure including two input and seven output parameters. The input parameters are pressure $(P)$ and temperature $(T)$, while the output parameters are density $\left(D, \mathrm{~g} / \mathrm{cm}^{3}\right)$, sound speed $(a, \mathrm{~m} / \mathrm{s})$, viscosity $\left(\mu, \mathrm{Pa}^{*} \mathrm{~s}\right)$, thermal conductivity $(\mathrm{k}, \mathrm{W} / \mathrm{m} * \mathrm{~K})$, specific heat at constant pressure $\left(C_{p}, \mathrm{~J} / \mathrm{g} * \mathrm{~K}\right)$, gamma $(\gamma)$ number and Prandtl number $\left(P_{r}\right)$. According to the working conditions of the cooling channels of the scramjet, the training databases were generated by using the interactive SuperTrapp database with the input temperature varying from $300 \mathrm{~K}$ to $1000 \mathrm{~K}$, and the input pressure varying from $2.5 \mathrm{MPa}$ to $6.0 \mathrm{MPa}$. Three ANN training databases are built respectively for the aforementioned three kerosene surrogate models, i.e., Fan's three-component model [4], Cheng's five-component model [5] and Zhong's ten-component model [6]. The prediction accuracy of the trained ANN will be validated against the exact ECS predictions calculated by SuperTrapp. 


\section{Artificial neural network and training}

\section{A. Algorithm of BP-ANN}

In this study, a classical back-propagation artificial neural network (BP-ANN) was used to model the thermal properties of the China RP3 kerosene at supercritical pressures. In this following part, the algorithm of a BP-ANN structure will be introduced detailedly.

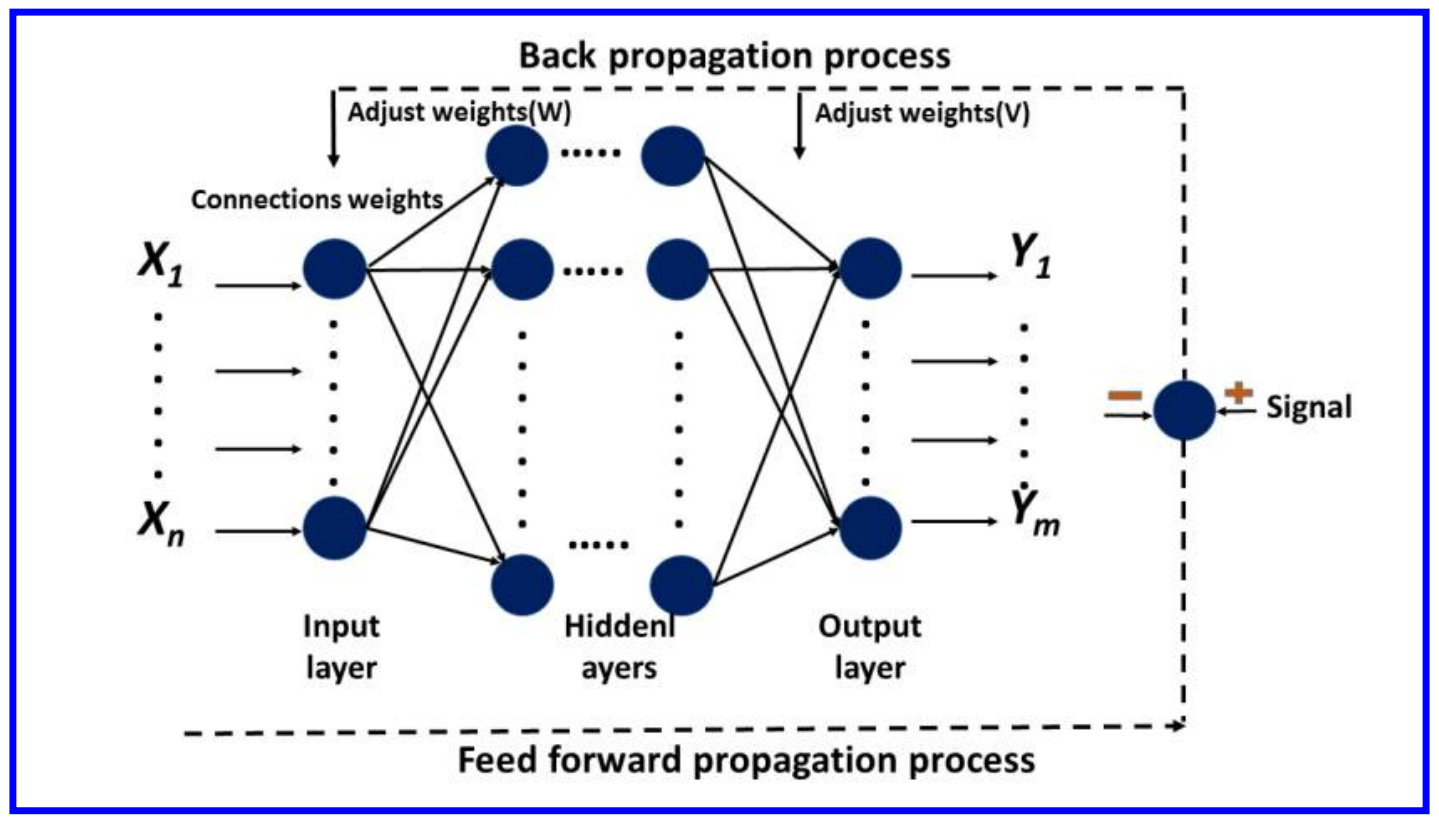

Figure 1.Schematic diagram of three-layer BP-ANN

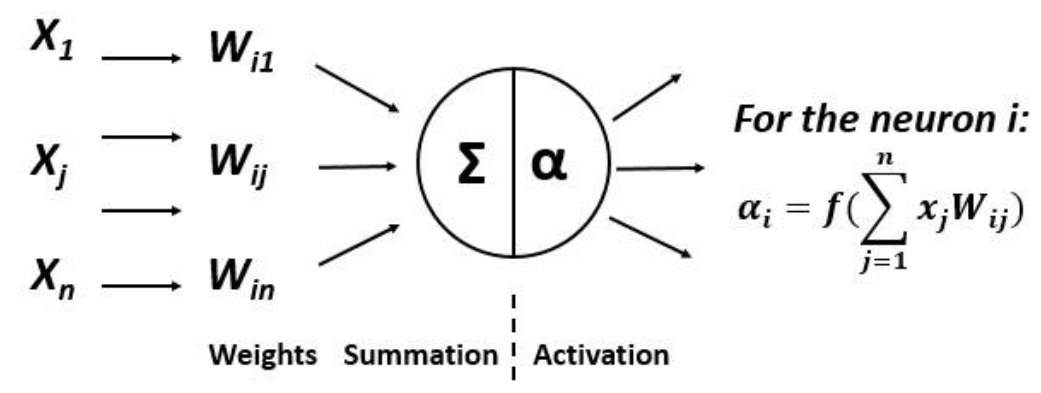

Figure 2. Information processing in a single neural-network neuron.

The basic building block of ANN is the neuron which like a "black box" consisted of weighted inputs and an output. Figure 2 illustrates how signals are processed through a single neuron. First, the neuron receives weighted activations of other neurons through its incoming connections and added up. The summation is then passed through an activation function $f$, the outcome $\alpha$ being the new activation which is multiplied by the specific weight and transferred to the next layer's neurons. Usually, a logistic sigmoid activation function is used and given by:

$$
f(z)=\frac{1}{1+e^{-z_{n}}}
$$

Where $z_{n}=\sum_{j=1}^{n} x_{j} W_{i j}$ is the weighted sum of the input activation for each neuron.

As shown in Figure 1, a typical multilayer BP-ANN architecture usually consists of an input layer, some hidden layers and an output layer and each layer is composed of simple neurons operating in parallel. Once the number of hidden layers and the number of neurons in each hidden layer were determined, the connection weights between the 
neurons were adjusted by information transmitting forward and errors transferring reversely during the process of training, It means that any errors made by the network during training get sent backwards through it in an attempt to correct it and so teach the network what is right and wrong, just as Figure 1 shown. And during the learning processer, the error is estimated by the mean squared error (MSE), defined as:

$$
M S E=\frac{1}{n} \sum_{i=1}^{n}\left(y_{i}-t_{i}\right)^{2}
$$

Where $y_{i}, n$ is the network output value and total number respectively, $t_{i}$ is the target value, and smaller MSE values indicate better ANN performance. When the MSE reaches a minimum or the steps of training reach a maximum, network training is considered complete and the weights are stored.

During backpropagation, the weights were updated according to the gradient descent algorithm which has been detailedly introduced in many articles [19-21]. The change of weight $W_{i j}$ in the connection neuron $i$ to $j$ can be written as:

$$
\Delta W_{i j}=-l_{r} \frac{\partial M S E}{\partial W_{i j}}
$$

where $l_{r}$ is the learning rate which is very important since a high value can cause too strong a change, causing the minimum to be missed, while a too low learning rate slows the training unnecessarily. In order to minimize the error function $M S E, W_{i j}$ needs to be decreased when $\frac{\partial M S E}{\partial W_{i j}}>0$, and $W_{i j}$ needs to be increased when $\frac{\partial M S E}{\partial W_{i j}}<0$. In order to avoid oscillation inside the network such as alternating connection weights, and to improve the rate of convergence, an adaptive learning rate was adopted[22]. When according to time continuous, similar to a ball rolling down a mountain, whose current speed is determined not only by the current slope of the mountain but also by its own inertia (momentum), so the change of weight $W_{i j}$ at time $(\mathrm{t}+1)$ is $\Delta W_{i j}(t+1)$, written as:

$$
\Delta W_{i j}(t+1)=(\mathrm{m}-1) l_{r} \frac{\partial M S E}{\partial W_{i j}}+m \Delta W_{i j}(t)
$$

Where $m$ is the momentum in $[0,1]$. Momentum is not only a technique to keep the weights changing in the direction of the previous step to speed up the convergence, it also has the benefit of avoiding the abrupt changing in the opposite direction when the direction change. Because the influence of the momentum in the weight update can become too large, a momentum adaptation technique was developed to use in parallel with the learning rate adaptation[23].

\section{B. Databases acquisition and Preprocessing}

This study aims to develop three ANN models which can efficiently and accurately estimate the properties of the aforementioned three surrogate models of China RP-3 kerosene at supercritical pressures. It is almost impossible to get sufficient databases of the cooling channel for scramjet applications based on experiment, where typical pressure is approximately 2.6-6 Mpa and temperature varies from $300 \mathrm{~K}$ to $1000 \mathrm{~K}$. But reliable data is one of the most important decisions in the development of the ANN models. Accordingly, the training databases (2.5 Mpa $\leq \mathrm{P} \leq 6 \mathrm{Mpa}$, $300 \mathrm{~K} \leq \mathrm{T} \leq 100 \mathrm{~K}$ ) were established by calling the SuperTrapp program. It is difficult to select the lowest number of data points to train the ANN because a lacked database can't fully describe the characteristics of the cases while an excess one will slow down the training efficiency. Considering the characteristics of the properties of China RP-3 kerosene at supercritical pressure and applying trial and error method, the most suitable training database for each surrogate model is established as shown in Table 1 . The pressure increment $(\Delta \mathrm{P})$ is a constant of $0.1 \mathrm{Mpa}$, and the temperature increments $(\Delta \mathrm{T})$ in each range are varied as in Table 1 . The volume of each training database is 49086.

Table 1. Training database for each surrogate model of kerosen

\begin{tabular}{ccccc}
\hline Kerosene & $\mathrm{P}(\mathrm{Mpa})$ & $\mathrm{T}(\mathrm{K})$ & $\Delta \mathrm{T}(\mathrm{K})$ & Volume \\
\hline \multirow{3}{*}{$3,5,10$-component } & & $300-600$ & 5 & 49086 \\
& $2.5-6$ & $600-700$ & 0.1 & 1 \\
\hline
\end{tabular}


In theory, ANN has no restriction on its training data despite the order of magnitude of the original sample varies greatly. But for higher training accuracy, it's better to put the samples at the same importance at the very beginning. So before the training, all the input and output variables are normalized to $[0,1]$, by the following equation:

$$
X^{\prime}=\frac{X-X_{\min }}{X_{\max }-X_{\min }}
$$

Where $X$ is the original value, $X^{\prime}$ is the normalized value, and $X_{\max }$ and $X_{\min }$ are the corresponding maximum and minimum of $X$.

After randomly disrupting the order of each training database, which volume is 49086 , it will be divided into two subsets: training set $95 \%$ and validation data set 5\%. During the training process, the training set is used to train the network, the validation set is used to validate the training results, and both training error and validation error are monitored. Some new data that is not included in the training database will be recalculated by SuperTrapp using the principle of ECS and will be used as the test set. The test set is used to obtain the overall accuracy of the trained ANN and to compare the performance of various ANN structures.

\section{Structure of ANN and Training}

Except for original databases and suitable preprocessing, another important decision in the development of ANN models is the network structure including the number of neurons of input layer and output layer, the number of hidden layers and the number of neurons in each layer. The number of neurons in the input layer and output layer is fixed by the input and output parameters. But it is difficult to choose the proper number of hidden layers and the suitable number of neuron in each one. Because the more complex of the ANN model, the better ANN can capture the nonlinear relationship of the input/output while the more difficult and slower to train it. By trial and error, it is found that a ANN model with three hidden layers and 30-50 neurons in each layer can produce good results in estimation of thermophysical properties of kerosene in supercritical pressures. Once the structure of the ANN model determined, results change obviously by varying the total training epochs. In order to choose the most appropriate number of neurons in each hidden layer and the suitable total training epochs, comparisons for each surrogate model of China RP-3 kerosene were conducted as shown in Table 2.

Table 2. The influence of neurons in each hidden layer and maximum training epochs

\begin{tabular}{ccccc}
\hline Kerosene & Neurons & Epochs & TrainingSetMSE & ValidationSetMSE \\
\hline 3-component & $(30,30,30)$ & 5000 & $1.25469 \mathrm{e}-06$ & 0.00126018 \\
3-component & $(30,30,30)$ & 1,0000 & $3.6607 \mathrm{e}-07$ & 0.0010434 \\
3-component & $(30,30,30)$ & 5,0000 & $1.24899 \mathrm{e}-07$ & 0.000574671 \\
3-component & $(30,30,30)$ & 10,0000 & $7.35931 \mathrm{e}-08$ & 0.000410932 \\
3-component & $(30,30,30)$ & 30,0000 & $3.7521 \mathrm{e}-08$ & 0.000283361 \\
5-component & $(30,30,30)$ & 5000 & $1.58614 \mathrm{e}-05$ & 0.00215845 \\
5-component & $(30,30,30)$ & 5,0000 & $1.85088 \mathrm{e}-07$ & 0.000678435 \\
5-component & $(30,30,30)$ & 10,0000 & $1.20108 \mathrm{e}-07$ & 0.0005886 \\
5-component & $(30,30,30)$ & 30,0000 & $1.16312 \mathrm{e}-07$ & 0.0005683 \\
5-component & $(50,50,50)$ & 10,0000 & $1.08614 \mathrm{e}-07$ & 0.00057345 \\
5-component & $(50,50,50)$ & 30,0000 & $6.52347 \mathrm{e}-08$ & 0.00049839 \\
10-componen & $(50,50,50)$ & 10,0000 & $6.41611 \mathrm{e}-08$ & 0.000505993 \\
10-componen & $(50,50,50)$ & 30,0000 & $3.66905 \mathrm{e}-08$ & 0.000381389 \\
\hline
\end{tabular}

Usually the smaller of the mean squared error of training and validation, the better of the result that ANN can predict. By analyzing the result of test set later, it is found that the order of magnitude of trainingSetMSE and validationSetMSE should be as small as $10^{-8}$ and $10^{-4}$ respectively. According to the Table 2, the best ANN structures and training epochs for each surrogate model of China RP-3 kerosene can be concluded in Table 3.

Table 3. The best ANN structures for each surrogate model of China RP-3 kerosene

\begin{tabular}{ccccc}
\hline Kerosene & Neurons & Epochs & TrainingSetMSE & ValidationSetMSE \\
\hline 3-component & $(30,30,30)$ & 300000 & $3.7521 \mathrm{e}-08$ & 0.000283361 \\
5-component & $(50,50,50)$ & 300000 & $6.52347 \mathrm{e}-08$ & 0.00049839 \\
10-component & $(50,50,50)$ & 300000 & $3.66905 \mathrm{e}-08$ & 0.000381389 \\
\hline
\end{tabular}




\section{Results and discussion}

In the above sections, three trained ANN models have been successfully developed for the prediction of China RP3 kerosene properties at supercritical pressures. The following sections will mainly illustrate the model accuracy and efficiency by comparing the ANN predictions with the ECS calculations by SuperTrapp.

\section{A. Results of the test sets}

In order to illustrate the correlation between the predicted results of the trained ANN models and the calculated values of ECS by SuperTrapp, six test sets for each surrogate model of China RP-3 kerosene were randomly selected. The properties, including density, sound speed, viscosity, thermal conductivity, specific heat at constant pressure, gamma number and Prandtl number are calculated by ANN and SuperTrapp respectively for the comparison. And for each surrogate model of kerosene, the six randomly chosen samples have not been used in the training databases. Table 4 lists the detailed information of the six test sets for each surrogate model of kerosene, and the volume of each test set.

Table 4. The randomly chosen six set samples for each surrogate model of kerosene

\begin{tabular}{cccc}
\hline Kerosene & $\mathrm{P}(\mathrm{Mpa})$ & $\mathrm{T}(\mathrm{K})$ & Volume \\
\hline 3-component & $2.55,3.17,3.76,4.23,4.84,5.96$ & & \\
5-component & $2.62,3.26,3.89,4.31,4.63,5.87$ & $300-1000, \Delta \mathrm{T}=1$ & 701 \\
10-component & $2.53,3.38,3.68,4.17,4.72,5.79$ & & \\
\hline
\end{tabular}

For a succinct description, the value of all the properties (including density, sound speed, viscosity, thermal conductivity, specific heat at constant pressure, gamma number and Prandtl number) is normalized to [0 1]. A comparison between the ANN predictions and the ECS calculations by SuperTrapp is shown from Figure 3 to Figure 5 .

It can be seen that the ANN predictions (the blue line ) match well with the ECS calculations by SuperTrapp(the symbol of square) for the three surrogate models of China RP-3 kerosene, even at the points with a significant change (around a lower pressure: 2.5-2.6 MPa).

Figure 6 illustrates the correlation coefficient (the value of R) between the ANN prediction and the ECS calculation by SuperTrapp at lower pressure where the properties with a more significant change. It can be seen that all the Rvalue is larger than 0.99 and approaching to 1 for each surrogate model. The data (x represents the calculation of ECS by SuperTrapp, y represents the prediction of ANN) matches well with the perfect fit ( $\mathrm{y}=\mathrm{x})$ and almost overlaps it. This demonstrates that the trained ANN models are accurate, valid and dependable in some extent.

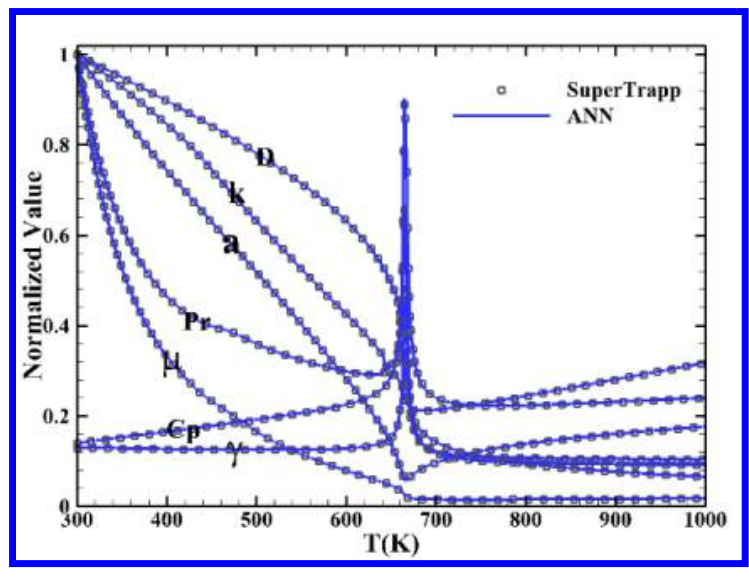

a)

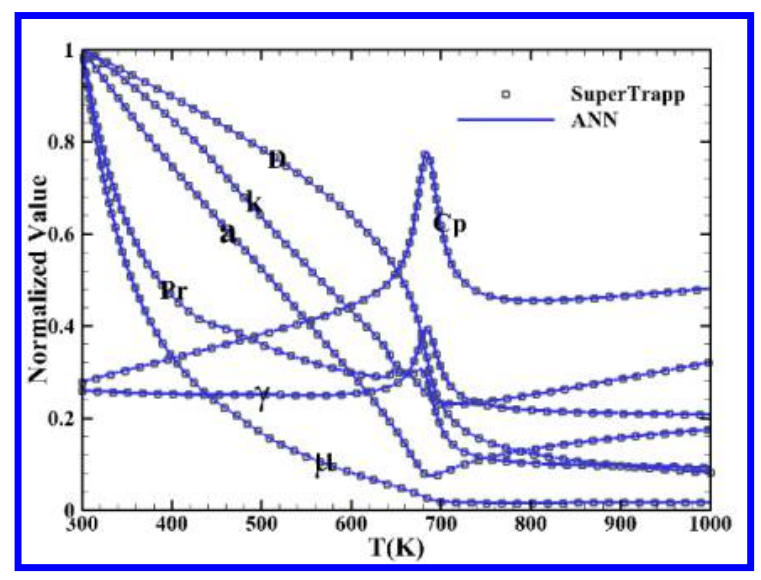

D) 

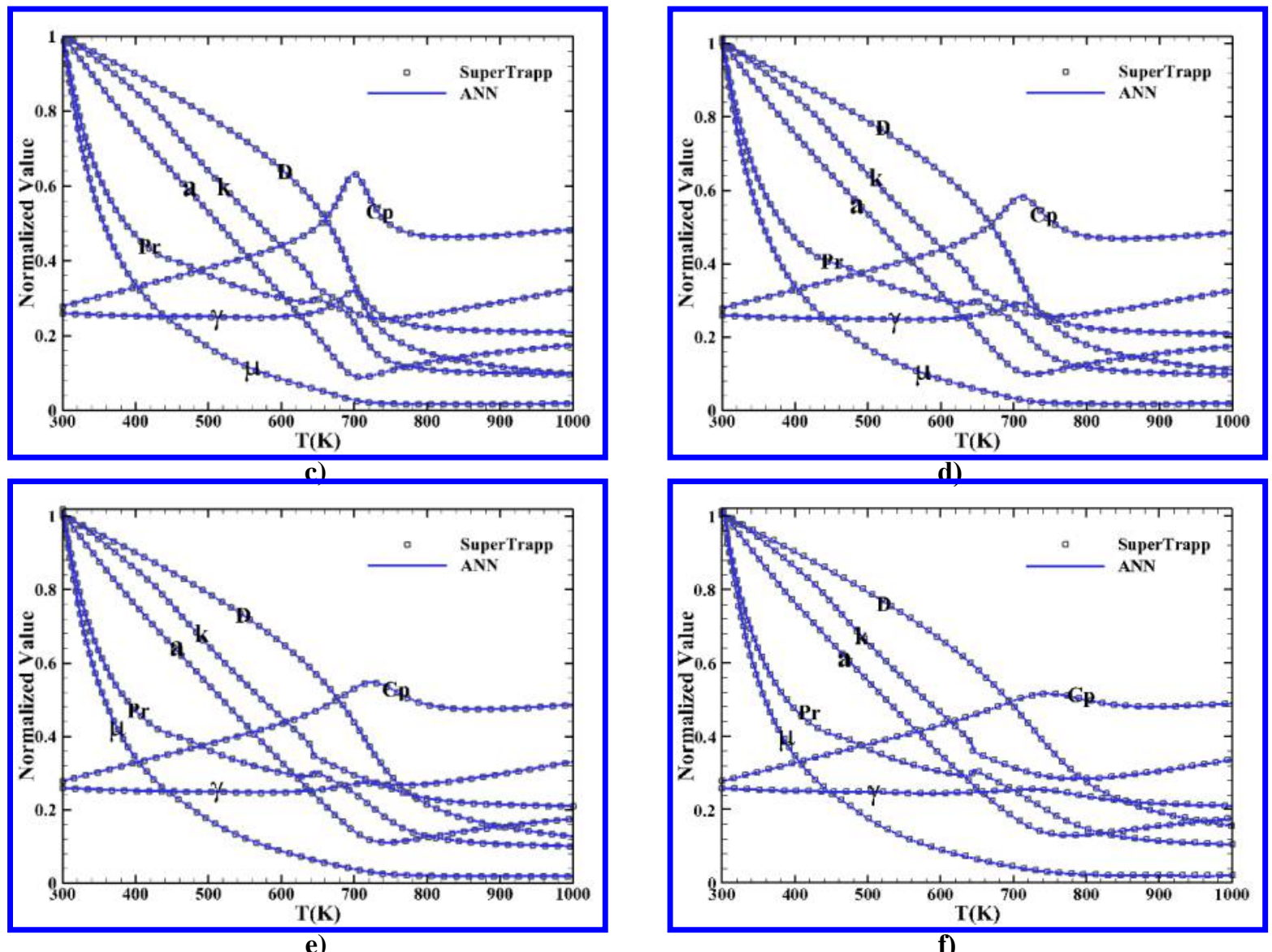

Figure 3. The comparison between ANN prediction and ECS calculation by SuperTrapp for the threecomponent surrogate model of China RP-3 kerosene, a) 2.55 MPa, b) 3.17 MPa, c) 3.76 MPa, d) 4.23 Mpa, e) 4.84 MPa, f) 5.91 Mpa.
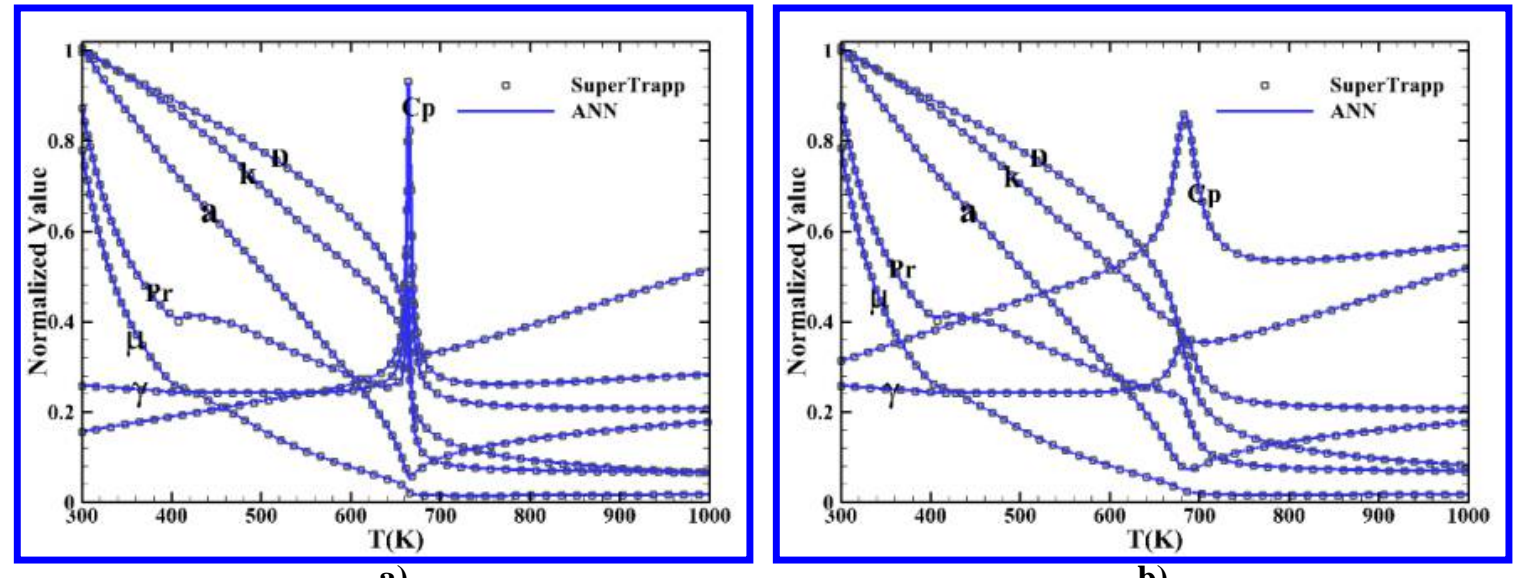

a)

b) 

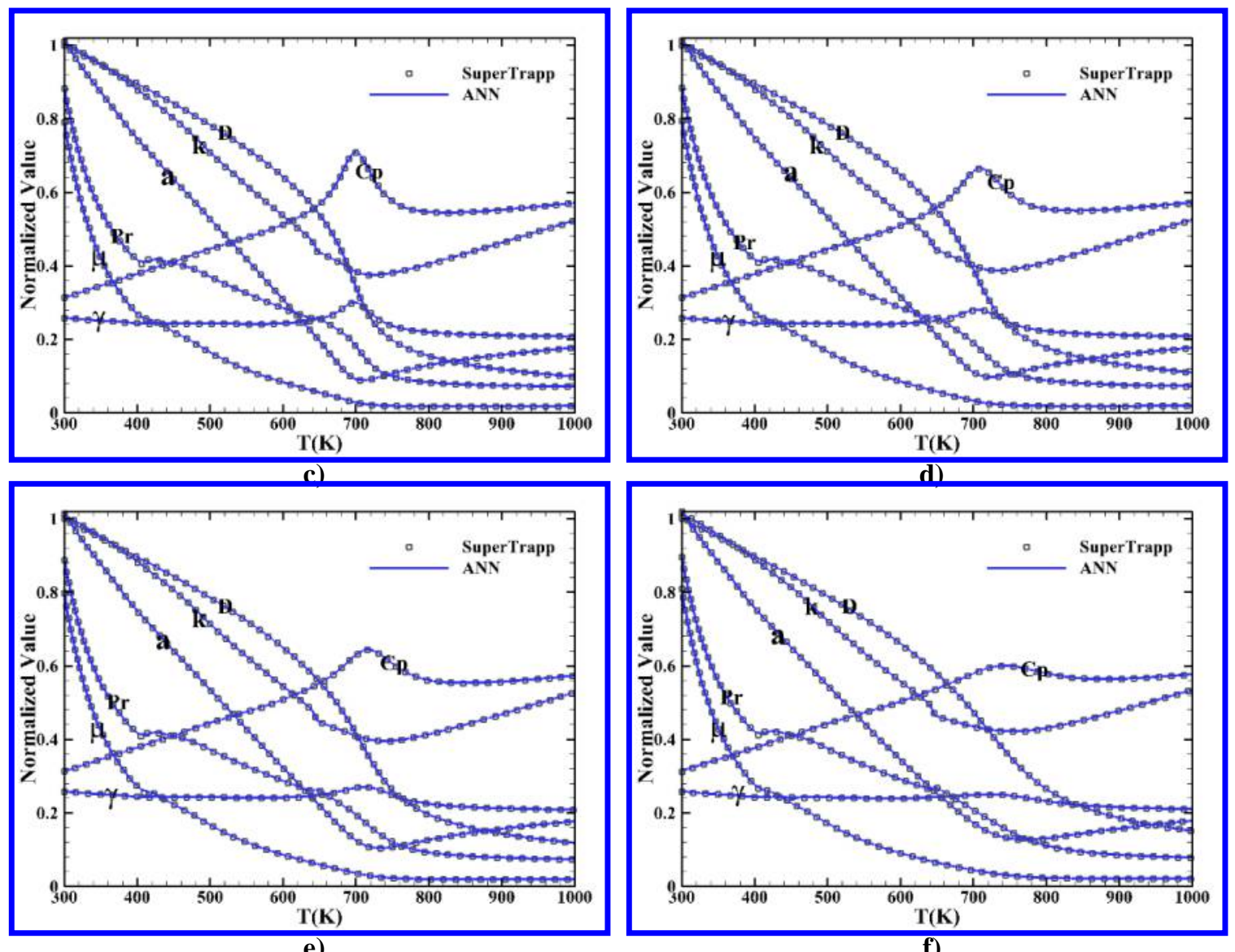

Figure 4. The comparison between ANN prediction and ECS calculation by SuperTrapp for the five-component surrogate model of China RP-3 kerosene, a) 2.62 MPa, b) 3.26 MPa, c) 3.89MPa, d) 4.31 Mpa, e) 4.63 MPa, f) 5.87 Mpa.

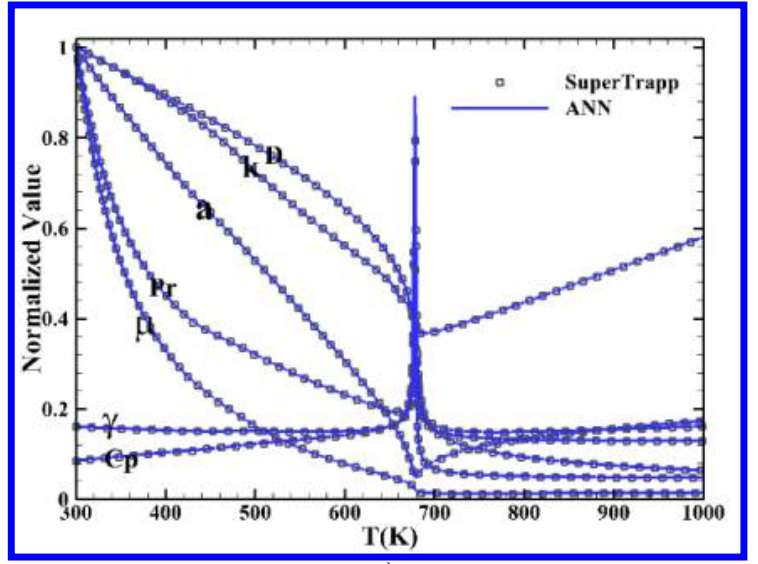

a)

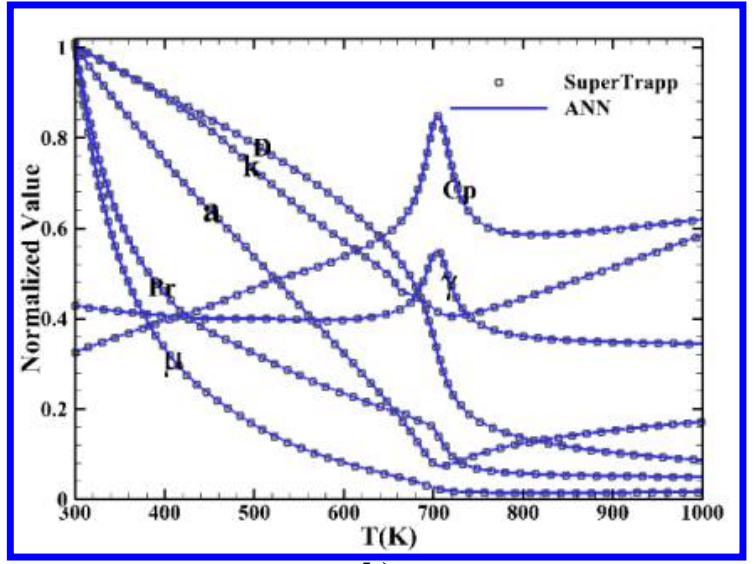

b) 

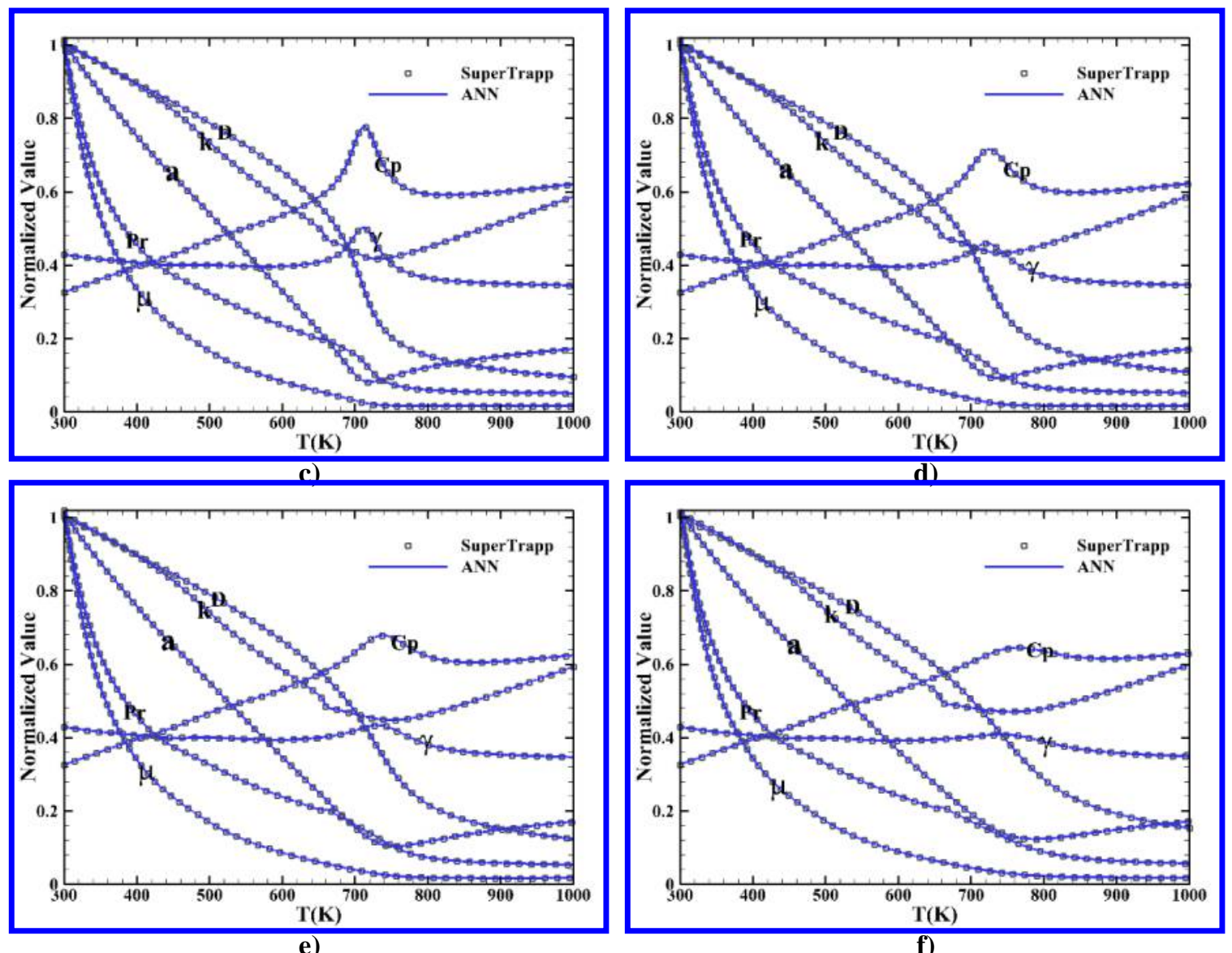

Figure 5. The comparison between ANN prediction and ECS calculation by SuperTrapp for the tencomponent surrogate model of China RP-3 kerosene, a) $2.53 \mathrm{MPa}$, b) 3.38 MPa, c) 3.68MPa, d) 4.17 Mpa, e) 4.72 MPa, f) 5.79 Mpa.

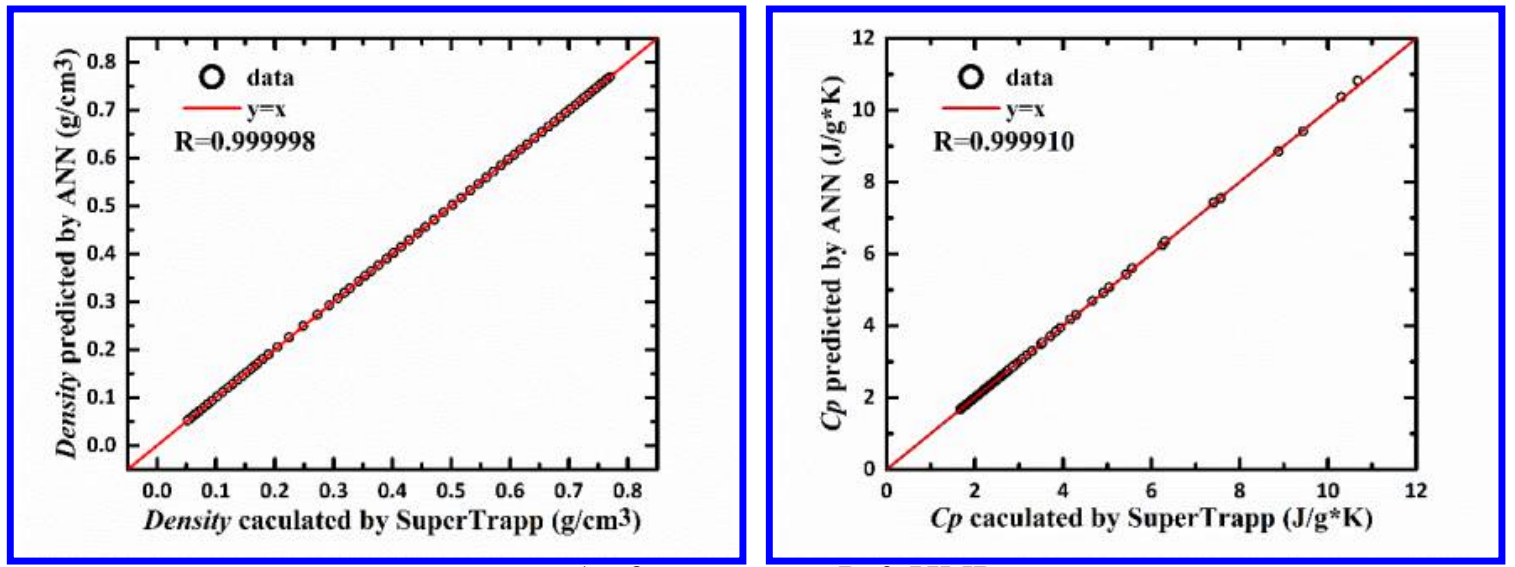

a) 3-component, $\mathrm{P}=2.55 \mathrm{MPa}$ 

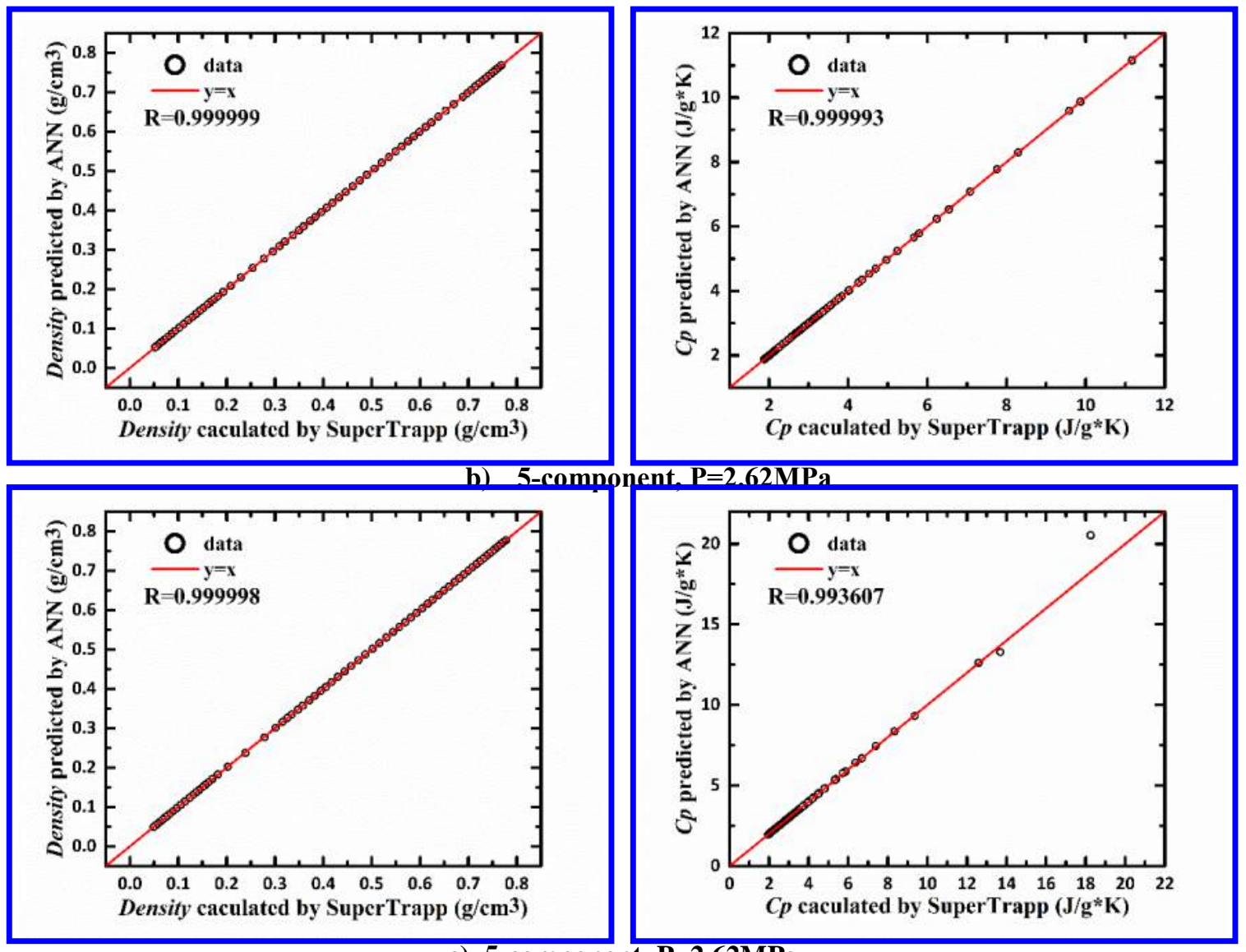

c) 5-component, $\mathrm{P}=2.62 \mathrm{MPa}$

Figure 6. The Correlation between ECS calculation by SuperTrapp and ANN prediction of density

and $C p$.

\section{B. Error analysis}

An comprehensive error analysis in the full pressure range of 2.5-6.0 Mpa and the temperature range of 300-1000 $\mathrm{K}$ is conducted to further validate the reliability and accuracy of the trained BP-ANN models. In this section, both the training data that have been used to develop the ANN models and other amount of new data that hasn't been used are assembled as a new testing set and used for error analysis, shown in Table 5.

Table 5. The data used to error analysis.

\begin{tabular}{cccc}
\hline Kerosene & $\mathrm{P}(\mathrm{Mpa})$ & $\mathrm{T}(\mathrm{K})$ & Volume of each set \\
\hline $3,5,10$ & $2.5-6.0, \Delta \mathrm{P}=0.05$ & $300-1000, \Delta \mathrm{T}=0.01$ & 497071 \\
\hline
\end{tabular}

For each set in Table 5, the fluid properties will be predicted by the trained ANN models and calculated by SuperTrapp respectively. The relative error of each property between ANN prediction and ECS calculation by SuperTrapp is defined as:

$$
\text { Error }=\left|\frac{\text { ANN }- \text { SuperTrapp }}{\text { SuperTrapp }}\right| * 100 \%
$$

Both the properties' relative error and its distribution $(0<$ error $\leqslant 0.1 \%, 0.1 \%<$ error $\leqslant 0.2 \%, 0.2 \%<$ error $\leqslant 0.5 \%$ and error $>0.5 \%$ ) were shown from figure 6 to figure 8 . And it can be seen that among the three surrogate models of kerosene, the maximum relative error for all properties is smaller than $3 \%$ within the targeted pressure (2.5-6.0Mpa) and temperature ranges $(300-1000 \mathrm{~K})$. Especially, when the pressure range is $2.7-6.0 \mathrm{MPa}$, the maximum relative error is even lower than $1 \%$ for most properties. While the large errors are mainly existing in the narrow pressure range of 
2.5-2.65 MPa, because the properties can change significantly when approaching the critical point (2.4-2.5 MPa). And observing the chart of bars from figure 6 to figure 8 , it can be concluded that the most relative errors are smaller than $0.1 \%$ and the relative errors exceeding $0.5 \%$ are far less than $1 \%$.

Comparing the results of the three trained ANN models from figure 6 to figure 8 , it can be found that the more complex of the alternative model of China RP-3 kerosene, the higher of the proportion of relative errors exceeding $0.5 \%$. And the finding can be further proved by the MSE for each surrogate model with the new testing set. The results were shown in table 6, in which the maximum and minimum MSE belongs to the ten-component and five-component surrogate model respectively.

Table 6. The testing MSE for each surrogate model of China RP-3 kerosene

\begin{tabular}{cc}
\hline Kerosene & MSE \\
\hline 3-component & $1.98197 \mathrm{e}-006$ \\
5-component & $8.97799 \mathrm{e}-006$ \\
10-component & $1.57077 \mathrm{e}-005$ \\
\hline
\end{tabular}
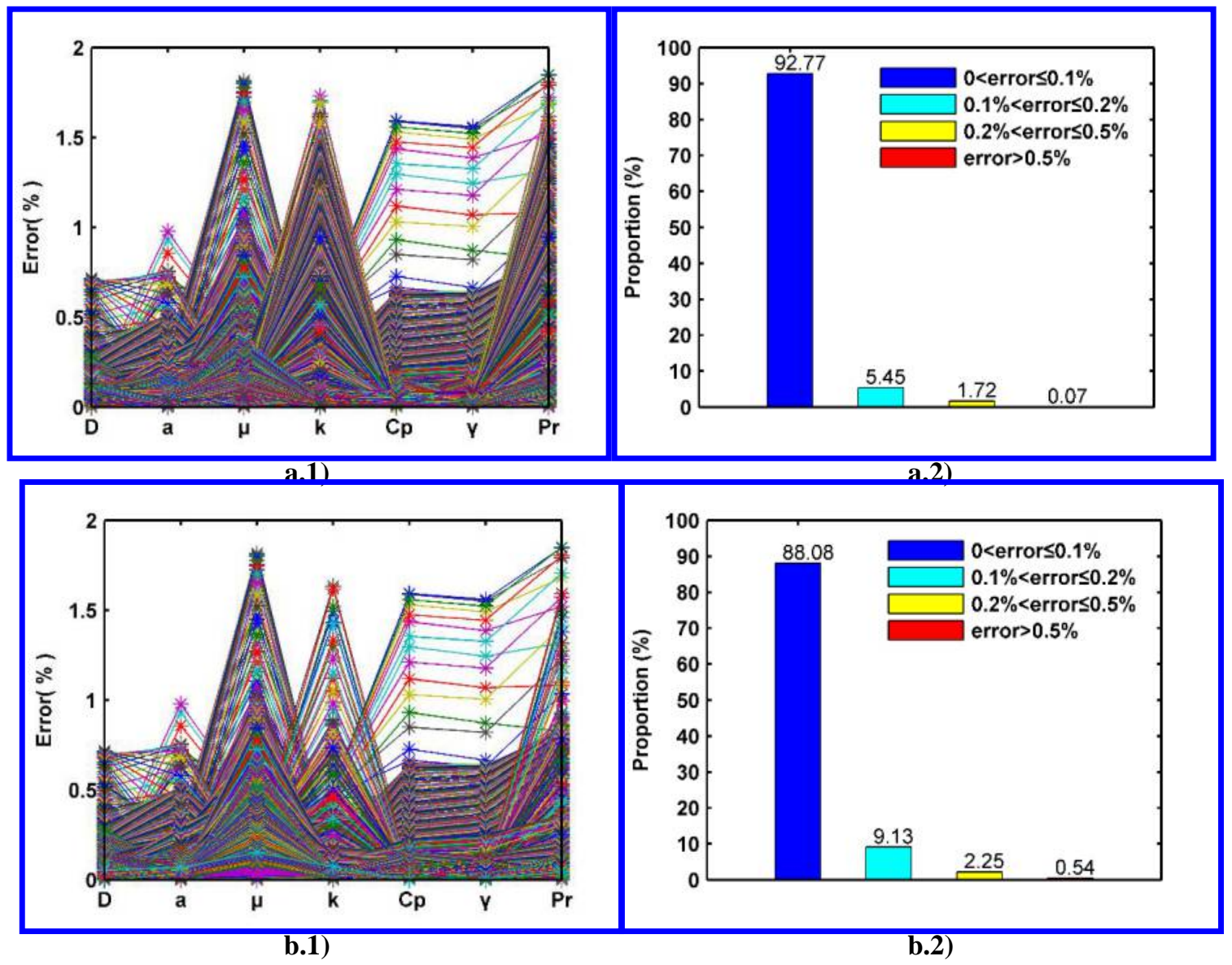


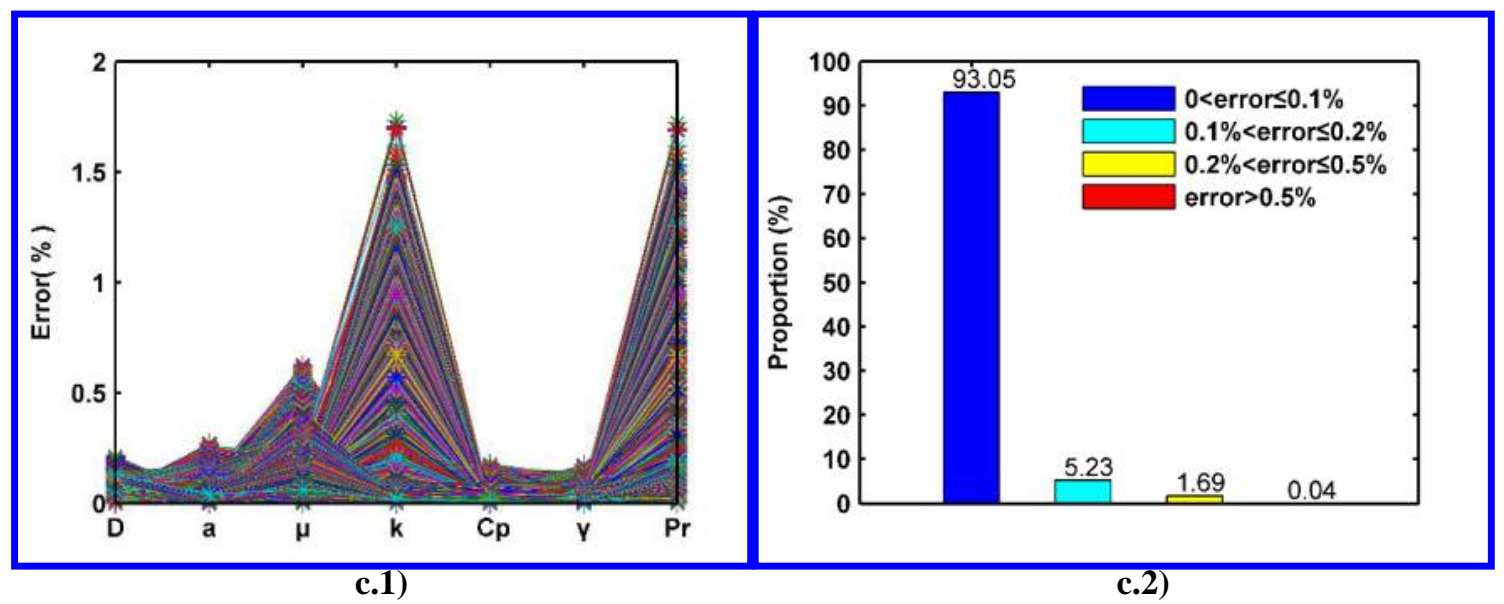

Figure 7. The properties' relative error and its distribution for the three-component surrogate model of kerosene, a) testing sets in 2.5-6.0 MPa, b) testing sets in 2.5-2.6 MPa, c) testing sets in 2.76.0 Mpa.
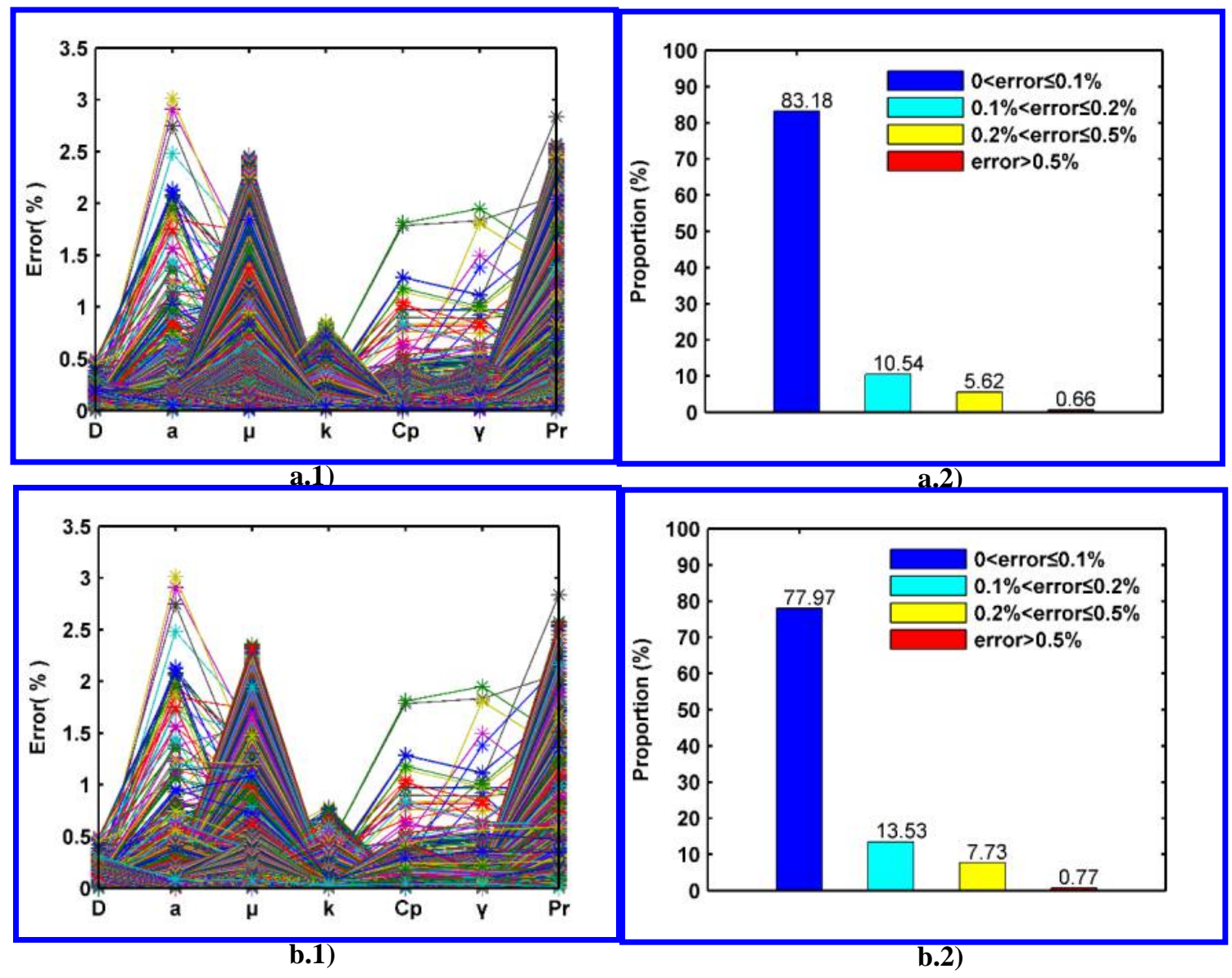

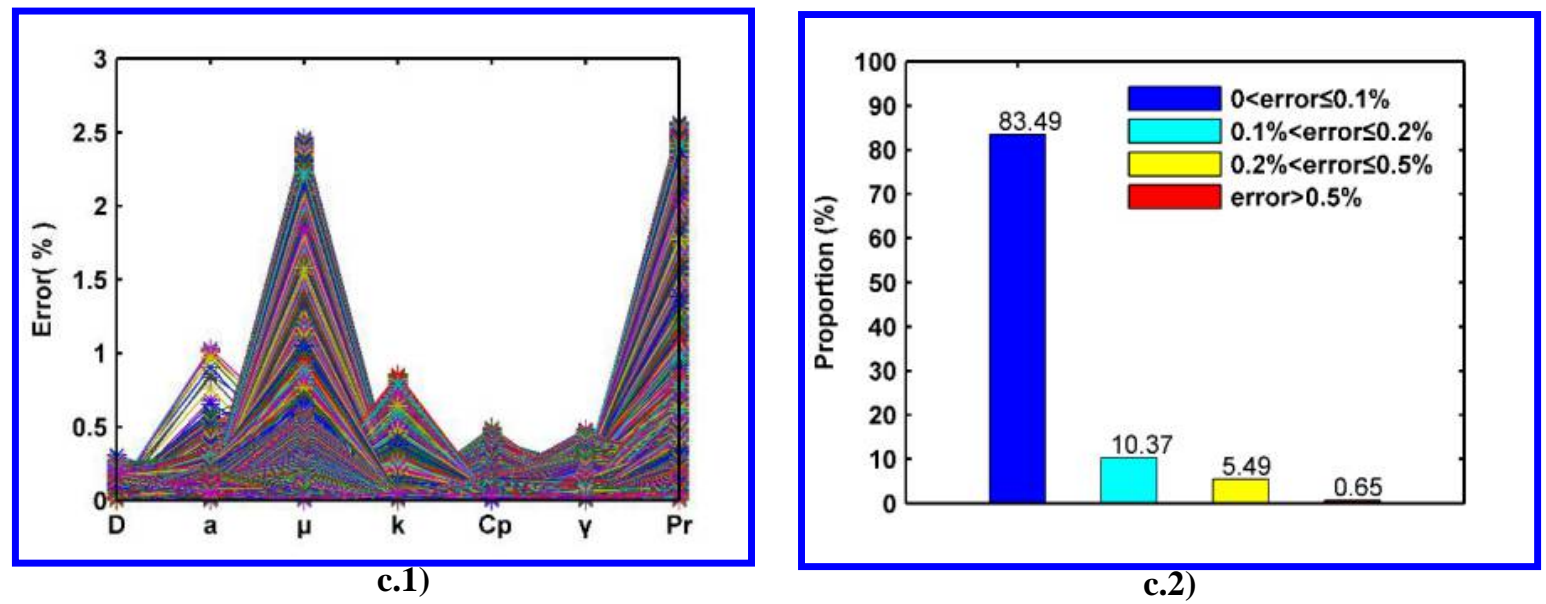

Figure 8. The properties' relative error and its distribution for the three-component surrogate model of kerosene, a) testing sets in 2.5-6.0 MPa, b) testing sets in 2.5-2.6MPa, c) testing sets in 2.76.0 Mpa.
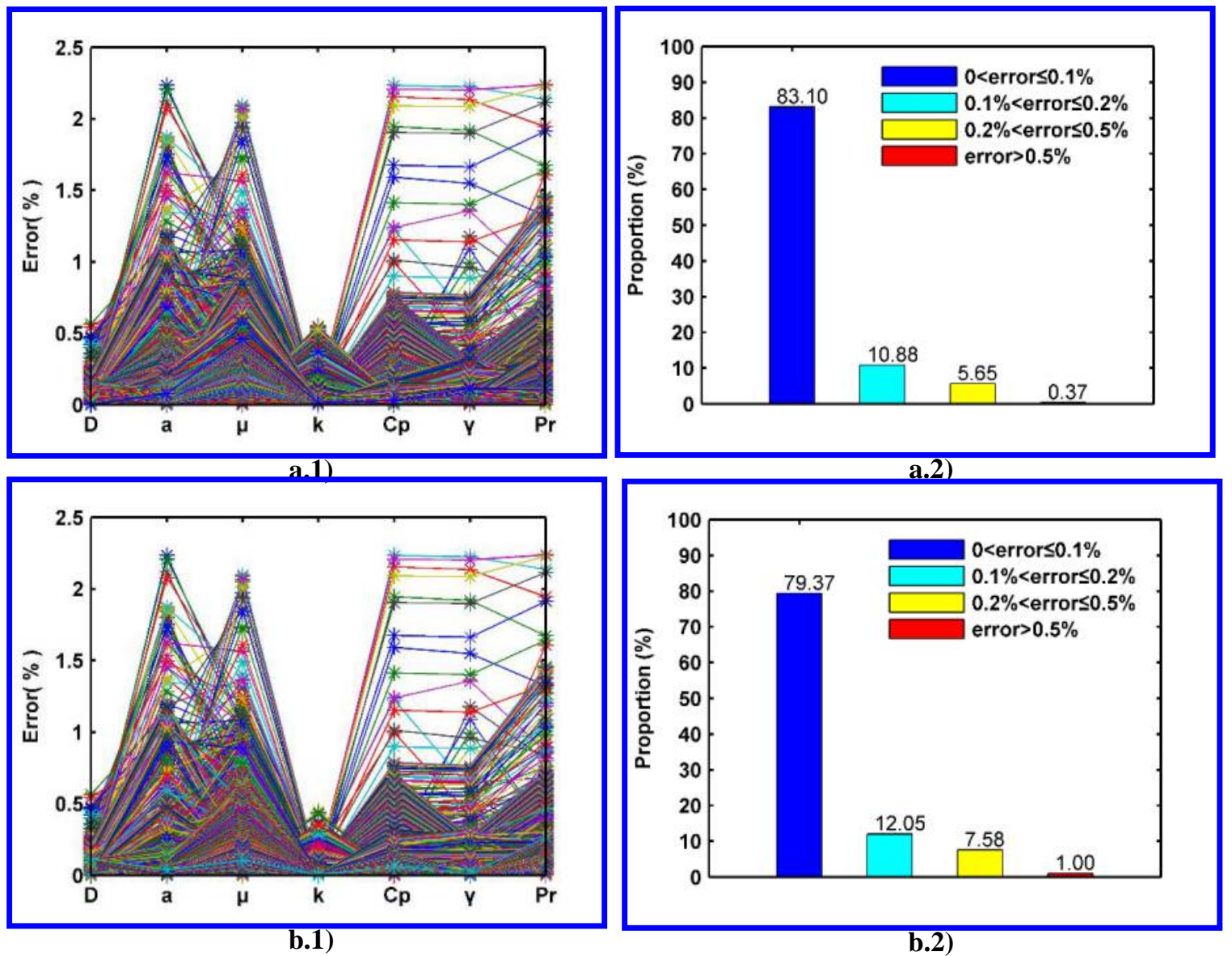


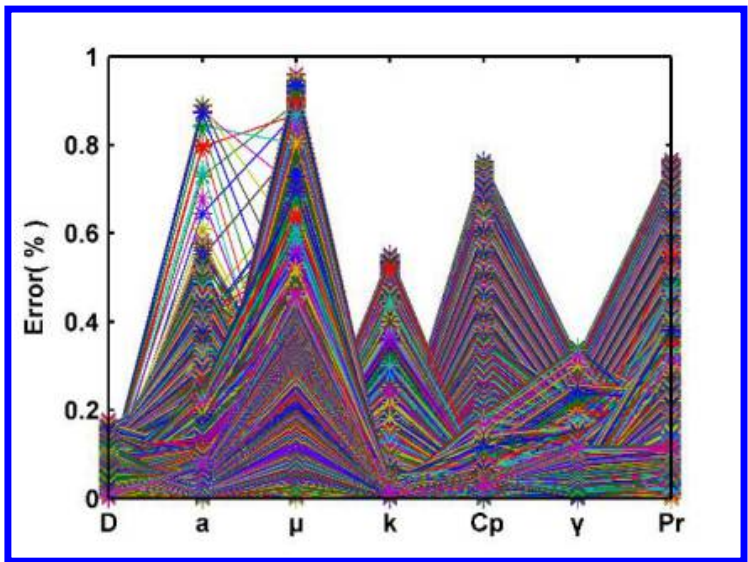

c.1)

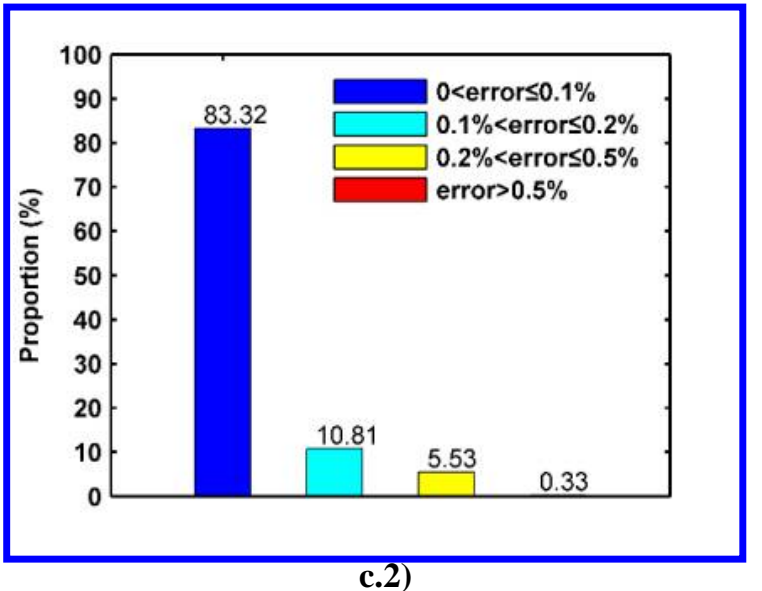

c.2)

Figure 9. The properties' relative error and its distribution for the three-component surrogate model of kerosene, a) testing sets in 2.5-6.0 MPa, b) testing sets in 2.5-2.6 MPa, c) testing sets in 2.76.0 Mpa.

\section{Efficiency analysis}

The computational efficiencies of the ANN prediction and the ECS calculation by SuperTrapp will be analyzed in this section. For a comprehensive explanation of the results, three sets were selected for each surrogate model, which includes the points in full range (volume is 25236), the points nearing the critical temperature (volume is 2211) and the points nearing the critical pressure and the critical temperature (volume is 8002), as Table 7 shown. And for each surrogate model of China RP-3 kerosene, the properties of the aforementioned three sets were predicted by the trained ANN models and calculated by ECS of SuperTrapp respectively, the computational time was listed in Table 7.

From Table 7, it can be seen that a trained ANN model can predict the fluid properties of China RP-3 kerosene at a speed several orders higher than the ECS calculation by SuperTrapp, especially near the critical point. Additionally, by comparing the results of the three surrogate model $(3,5,10$-component), it can be seen that the more complex of the surrogate model of kerosene, the longer of the computational time needed and the higher of the multiple that improved by ANN.

Table 7. Efficiency comparison between ANN prediction and ECS calculation by SuperTrapp

\begin{tabular}{|c|c|c|c|c|c|}
\hline Kerosene & Setting & Volume & $\begin{array}{c}\text { Time. } \\
\text { SuperTrapp }\end{array}$ & $\begin{array}{l}\text { Time. } \\
\text { ANN }\end{array}$ & $\frac{\text { Time. SuperTrapp }}{\text { Time. ANN }}$ \\
\hline \multirow{3}{*}{ 3-component } & $\begin{array}{c}2.5 \mathrm{MPa} \leqslant \mathrm{P} \leqslant 6.0 \mathrm{Mpa}, \\
\Delta \mathrm{P}=0.1 \mathrm{MPa} \\
300 \mathrm{~K} \leqslant \mathrm{~T} \leqslant 1000 \mathrm{~K} \\
\Delta \mathrm{T}=1 \mathrm{~K}\end{array}$ & 25236 & $682.21 \mathrm{~s}$ & $1.18 \mathrm{~s}$ & 578.14 \\
\hline & $\begin{array}{c}3.0 \mathrm{MPa} \leqslant \mathrm{P} \leqslant 4.0 \mathrm{Mpa}, \\
\Delta \mathrm{P}=0.1 \mathrm{MPa} \\
660 \mathrm{~K} \leqslant \mathrm{~T} \leqslant 680 \mathrm{~K}, \\
\Delta \mathrm{T}=0.1 \mathrm{~K}\end{array}$ & 2211 & $507.74 \mathrm{~s}$ & $0.11 \mathrm{~s}$ & 4615.79 \\
\hline & $\begin{array}{c}2.5 \mathrm{MPa} \leqslant \mathrm{P} \leqslant 2.6 \mathrm{Mpa}, \\
\Delta \mathrm{P}=0.1 \mathrm{MPa} \\
640 \mathrm{~K} \leqslant \mathrm{~T} \leqslant 680 \mathrm{~K} \\
\Delta \mathrm{T}=0.01 \mathrm{~K}\end{array}$ & 8002 & $6682.43 \mathrm{~s}$ & $0.41 \mathrm{~s}$ & 16298.61 \\
\hline 5-component & $\begin{array}{c}2.5 \mathrm{MPa} \leqslant \mathrm{P} \leqslant 6.0 \mathrm{Mpa}, \\
\Delta \mathrm{P}=0.1 \mathrm{MPa} \\
300 \mathrm{~K} \leqslant \mathrm{~T} \leqslant 1000 \mathrm{~K} \\
\Delta \mathrm{T}=1 \mathrm{~K}\end{array}$ & 25236 & $1193.28 \mathrm{~s}$ & $1.52 \mathrm{~s}$ & 785.05 \\
\hline
\end{tabular}



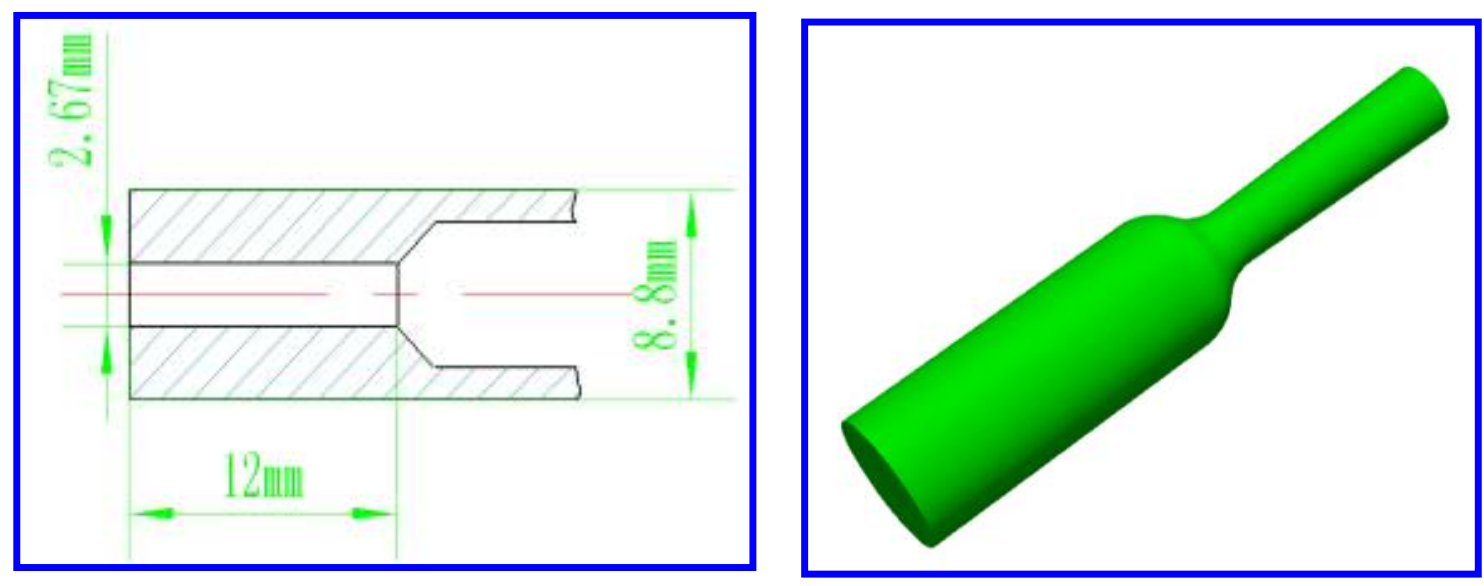

a)

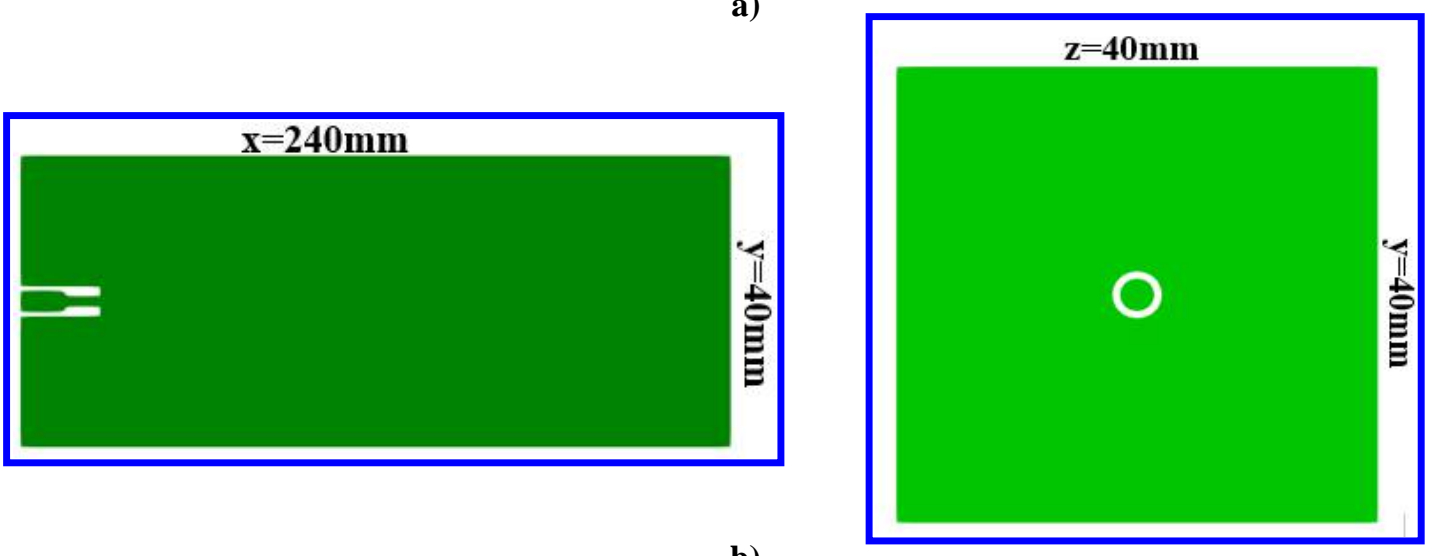

b)
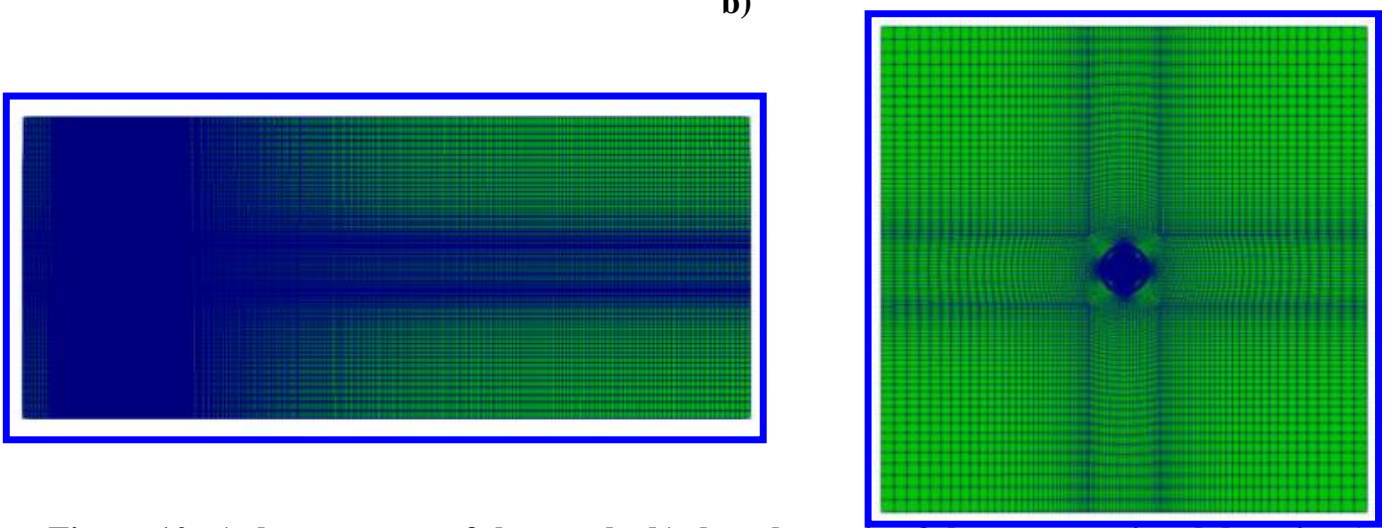

Figure 10. a) the structure of the nozzle, b) the schematic of the computational domain, c) the computational grids 


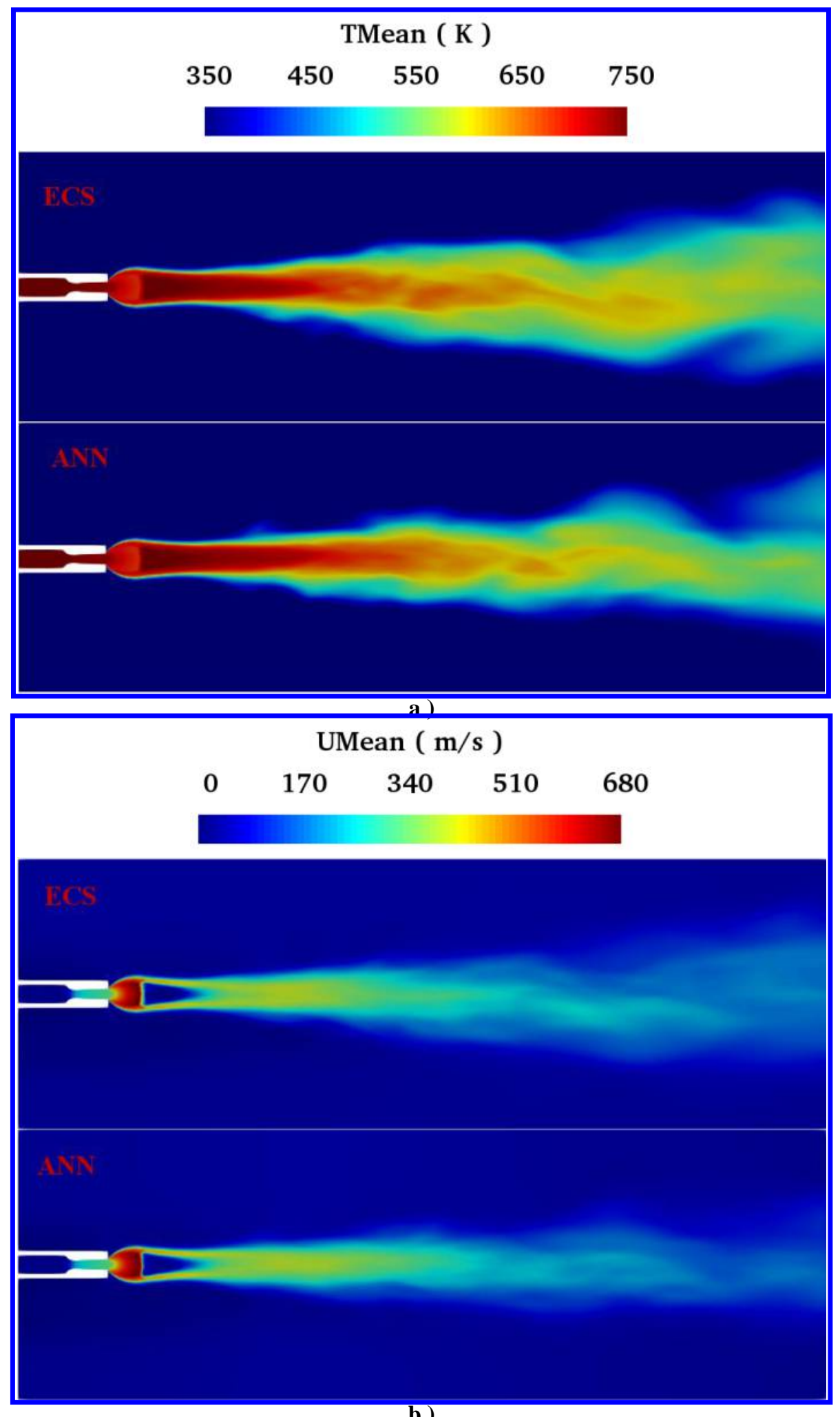

b )

Figure 11. The time-averaged contours of temperature and velocity, which were simulated by ANN and ECS respectively: a) the time-averaged contour of temperature, b) the time-averaged contour of velocity. 

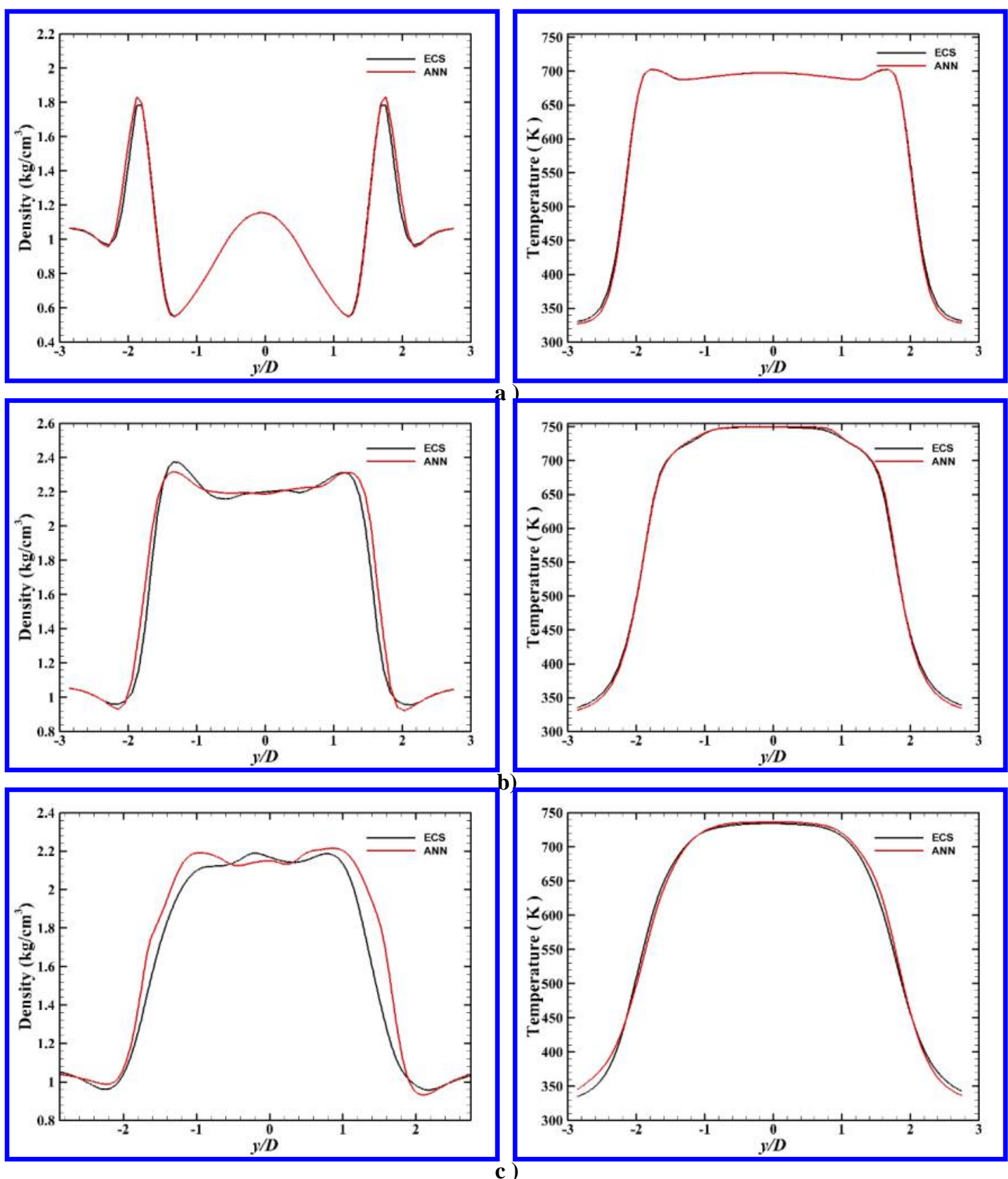


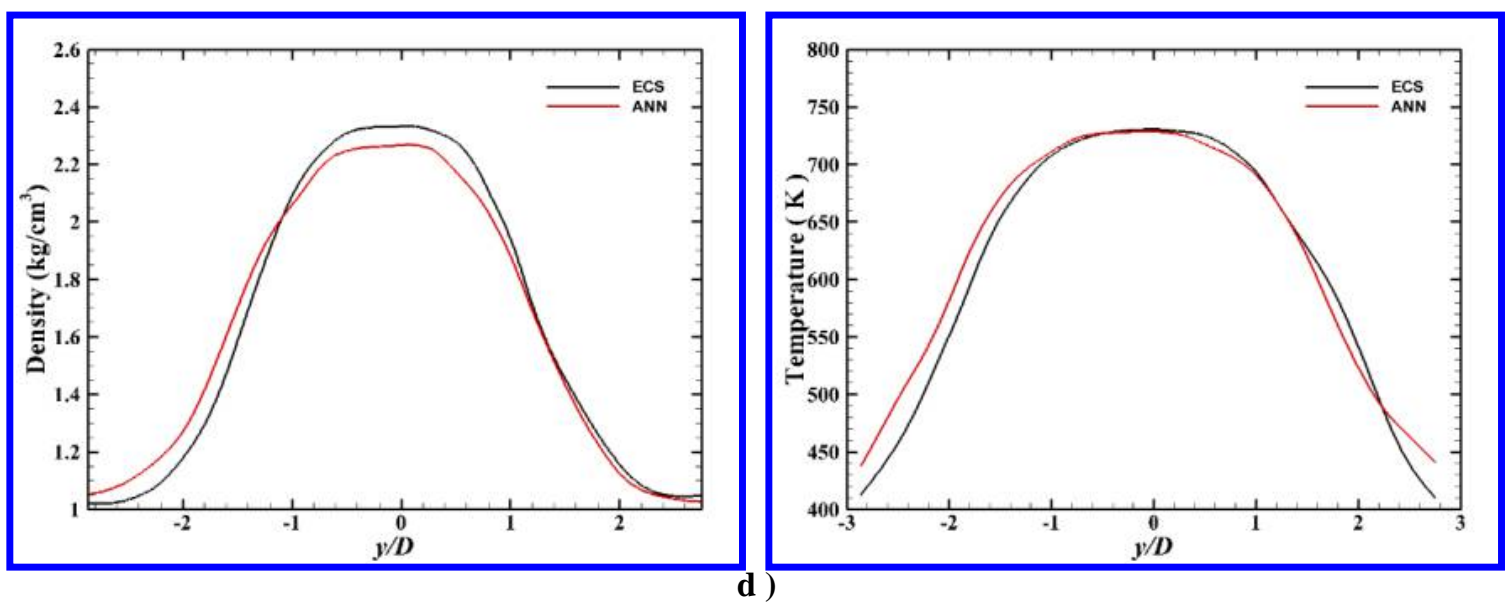

Figure 12. The comparison between ANN prediction and ECS calculation for density and temperature: a) $x=10 D$, b) $x=15 D, c) x=20 D, d$ ) $x=25 D$
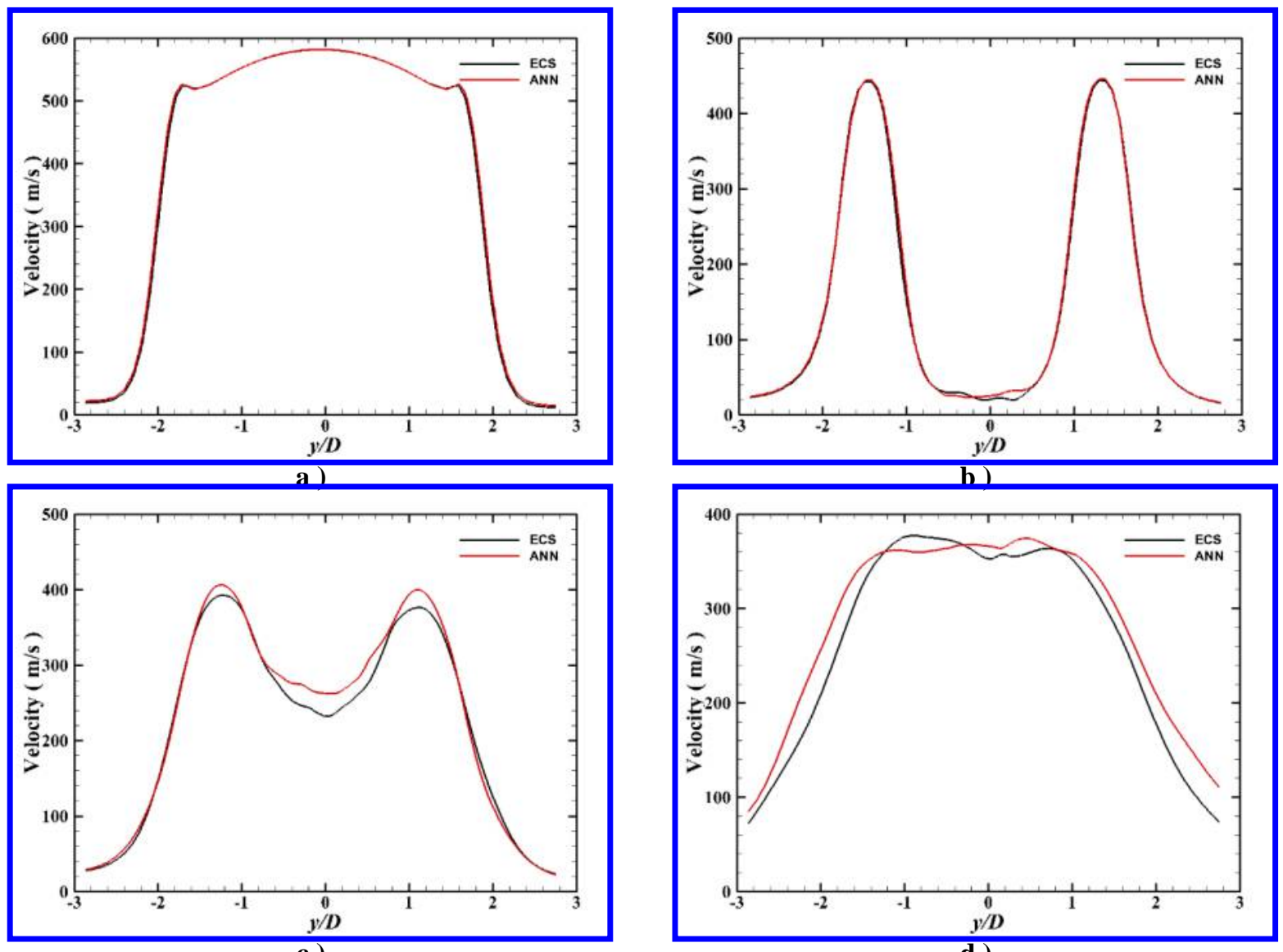

Figure 13. The comparison between ANN prediction and ECS calculation for velocity: a) $x=10 D, b) x=15 D$, c) $x=20 D, d) x=25 D$ 


\section{Conclusion}

In this study, the main object is to develop an efficient yet accurate method to predict the fluid properties of China RP3 kerosene at supercritical pressure by applying the artificial neural network (ANN) method, which would make CFD modeling coupled with real-gas effects computationally-affordable.

Based on the principle of Extended Corresponding State (ECS), three training databases were firstly built for each aforementioned surrogate model of kerosene by SuperTrapp respectively. Then, three ANN models have been successfully developed by adopting the back-propagation (BP) algorithm, adaptive learning rate and adaptive momentum method. And the best ANN structure with the lowest root mean square error (MSE) for each surrogate model of China RP3 kerosene was shown in Table 3, from which it can be seen that the biggest TrainingMSE and ValidationMSE is $6.52347 \mathrm{e}-08$ and 0.00049839 respectively among the three surrogate models, and the more complex the surrogate model is, the more complex the ANN structures will be.

Six samples were randomly selected as the test set for each surrogate model of kerosene and the properties were respectively estimated by ANN and SuperTrapp. The results of comparison show that the ANN predictions agree well with the ECS calculations by SuperTrapp even at the points approaching the critical pressure and critical temperature with a significant change. The analysis of the correlation coefficient (R) between the ANN prediction and ECS calculation by SuperTrapp at lower pressure show that all the R-value is bigger than 0.99 and approaching to 1 . Second, a comprehensive error analysis between ANN prediction and ECS calculation by SuperTrapp in full range has been conducted. And from the results of error analysis, it can be seen that the biggest relative error is lower than $3 \%$, the proportion of the relative error from 0 to $0.1 \%$ is higher than $80 \%$ and the relative error exceeding $0.5 \%$ is no more than $1 \%$. So it can be concluded that the three trained ANN models are accurate, valid and dependable. Then, the computational efficiency of the ANN and the ECS by SuperTrapp was compared, and the results show that a trained ANN model can predict the fluid properties of kerosene at a speed several orders (arriving at $10^{4}$ ) higher than the ECS calculation by SuperTrapp, especially near the critical point. In the last, the performance of the trained ANN coupling with CFD modellings was analyzed, and the results shows that the ANN models applied in CFD modelling are accurate and time-saving in some extend, but only the density was predicted by ANN in this study, which can't truly illustrate the efficiency of ANN coupling with CFD modellings.

ANN is an efficient and accurate method to predict the fluid properties of kerosene and the method can also be used to predict the properties of other substances. However, to truly reflect the computational efficiency when ANN is coupled with CFD, more properties such as thermal conductivity and viscosity should also be combinedly predicted by ANN in further studies.

\section{Acknowledgments}

The Project was supported by National Natural Science Foundation of China (Grant No. 11502270) and Training Program of the Major Research Plan of the National Natural Science Foundation of China (Grant No. 91641110). The authors are also grateful to National Supercomputer Center in Tianjin for providing the computational resource.

\section{References}

1. Zhong, F., Fan, X., Yu, G., Li, J., and Sung, C.-J. "Heat Transfer of Aviation Kerosene at Supercritical Conditions," Journal of Thermophysics and Heat Transfer Vol. 23, No. 3, 2009, pp. 543-550.

-2. Lander, H., and Nixon, A. C. "Endothermic fuels for hypersonic vehicles," Journal of Aircraft Vol. 8, No. 4, 1971, pp. 200-207.

3. Li, X., Huai, X., Cai, J., Zhong, F., Fan, X., and Guo, Z. "Convective heat transfer characteristics of China RP-3 aviation kerosene at supercritical pressure," Applied Thermal Engineering Vol. 31, No. 14-15, 2011, pp. 2360-2366.

4. Fan Xue-jun, Y. G. "Analysis of thermophysical properties of Daqing RP-3 aviation kerosene," Journal of Propulsion Technology Vol. vol.27, No. 2, 2006, pp. 187-192.

5. Di Cheng, X. W. a. X. F. "ThermoPhysical Surrogate Modelling of RP-3 aviation kerosene," The Seventh National Fluid Mechanics Conference. GuiLin, China, December 2012.

-6. Zhong, F.-Q., Fan, X.-J., Wang, J., Yu, G., and Li, J.-G. "Characteristics of compressible flow of supercritical kerosene," Acta Mechanica Sinica Vol. 28, No. 1, 2012, pp. 8-13.

7. "Corresponding States Principle Using Shape Factors."

-8. Queimada, A. J., Stenby, E. H., Marrucho, I. M., and Coutinho, J. A. P. "A new Corresponding States model for the estimation of thermophysical properties of long chain n-alkanes," Fluid Phase Equilibria Vol. 212, No. 1-2, 2003, pp. 303-314. 
9. Huber, M. L. "NIST Thermophysics Properties of Hydrocarbon Mixtures Database (SUPERTRAPP)",. Gaithersburg: National Institute of Standards and Technology Standard Reference Data Program, 2007.

-10. Sen, B., and Menon, S. "Representation of Chemical Kinetics by Artificial Neural Networks for Large Eddy Simulations," AIAA/ASME/SAE/ASEE Joint Propulsion Conference \& Exhibit/43rd, 2007.

-11. Kalogirou, S. A. "Applications of artificial neural-networks for energy systems.," Applied Energy Vol. 67, 2000, pp. 17-35.

12. SSencan, A., and Kalogirou, S. A. "A new approach using artificial neural networks for determination of the thermodynamic properties of fluid couples," Energy Conversion and Management Vol. 46, No. 15-16, 2005, pp. 2405-2418.

13. $\mathrm{Li}, \mathrm{C}$., and $\mathrm{Yu}, \mathrm{C}$. "Performance evaluation of public non-profit hospitals using a BP artificial neural network: the case of Hubei Province in China," Int J Environ Res Public Health Vol. 10, No. 8, 2013, pp. 3619-33.

-14. Mora R, J. E., Pérez T, C., González N, F. F., and Ocampo D, J. D. D. "Thermodynamic properties of refrigerants using artificial neural networks," International Journal of Refrigeration Vol. 46, 2014, pp. 9-16.

15. Longo, G. A., Zilio, C., Ceseracciu, E., and Reggiani, M. "Application of Artificial Neural Network (ANN) for the prediction of thermal conductivity of oxide-water nanofluids," Nano Energy Vol. 1, No. 2, 2012, pp. 290-296.

16. Zhao, N., and Li, Z. "Experiment and Artificial Neural Network Prediction of Thermal Conductivity and Viscosity for Alumina-Water Nanofluids," Materials (Basel) Vol. 10, No. 5, 2017.

17. Kapoor, R., Sankaran, V., and Menon, S. "Towards Engineering Les of Reacting Flows:Artificial Neural Networks for Efficient Kinetics Modeling," 2003.

18. Choi, Y., and Chen, J. Y. "Fast prediction of start-of-combustion in HCCI with combined artificial neural networks and ignition delay model," Proceedings of the Combustion Institute Vol. 30, No. 2, 2005, pp. 2711 2718.

19. Rumelhart, D. E. H., Geoffrey E.; Williams, Ronald J. "Learning representations by back-propagating errors," Nature Vol. 323, 1986, pp. 533-536.

20. Schmidhuber, J. "Deep learning in neural networks: an overview," Neural Netw Vol. 61, 2015, pp. 85-117.

21. Eiji Mizutani, S. E. D. a. K. N. "On derivation of MLP backpropagation from the Kelley-Bryson optimalcontrol gradient formula and its application," Proceedings of the IEEE International Joint Conference on Neural Networks., 2000.

-22. Li, Y., Fu, Y., Li, H., and Zhang, S.-W. "The Improved Training Algorithm of Back Propagation Neural Network with Self-adaptive Learning Rate," 2009, pp. 73-76.

23. E.Fiesler, M. M. a. "Neural Networks with Adaptive Learning Rate and Momentum Terms.," IDIAP Technical report Vol. 95-04, 1995.

24. Yao, W., Lu, Y., Li, X., Wang, J., and Fan, X. "Improved Delayed Detached Eddy Simulation of a high-Ma regenerative-cooled scramjet combustor based on skeletal kerosene mechanism," 2016.

-25. Yao, W., Wang, J., Lu, Y., Li, X., and Fan, X. "Full-scale Detached Eddy Simulation of kerosene fueled scramjet combustor based on skeletal mechanism," 20th AIAA International Space Planes and Hypersonic Systems and Technologies Conference. Vol. AIAA 2015-3579, Glasgow, Scotland, 2015.

-26. Li, X., Yao, W., and Fan, X. "Large-Eddy Simulation of Time Evolution and Instability of Highly Underexpanded Sonic Jets," AIAA Journal Vol. 54, No. 10, 2016, pp. 3191-3211.

-27. Li, X., Zhou, R., Yao, W., and Fan, X. "Flow characteristic of highly underexpanded jets from various nozzle geometries," Applied Thermal Engineering Vol. 125, 2017, pp. 240-253. 